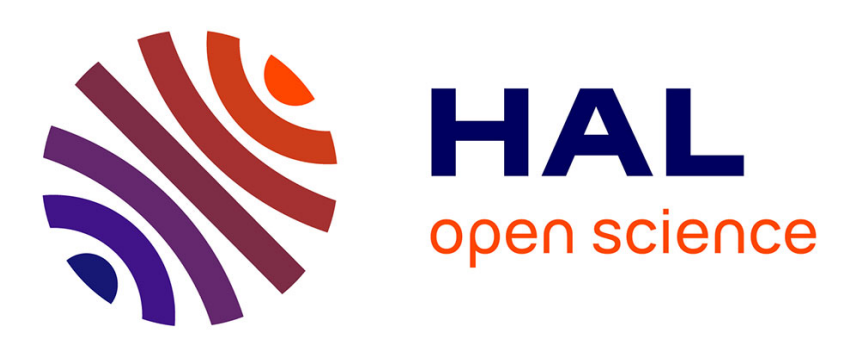

\title{
Structural-acoustic modeling of automotive vehicles in presence of uncertainties and experimental identification and validation
}

\author{
J.-F. Durand, Christian Soize, L. Gagliardini
}

\section{- To cite this version:}

J.-F. Durand, Christian Soize, L. Gagliardini. Structural-acoustic modeling of automotive vehicles in presence of uncertainties and experimental identification and validation. Journal of the Acoustical Society of America, 2008, 124 (3), pp.1513-1525. 10.1121/1.2953316 . hal-00685108

\section{HAL Id: hal-00685108 \\ https://hal.science/hal-00685108}

Submitted on 4 Apr 2012

HAL is a multi-disciplinary open access archive for the deposit and dissemination of scientific research documents, whether they are published or not. The documents may come from teaching and research institutions in France or abroad, or from public or private research centers.
L'archive ouverte pluridisciplinaire HAL, est destinée au dépôt et à la diffusion de documents scientifiques de niveau recherche, publiés ou non, émanant des établissements d'enseignement et de recherche français ou étrangers, des laboratoires publics ou privés. 
Structural-acoustic modeling of automotive vehicles in presence of uncertainties and experimental identification and validation

\author{
Jean-François Durand and Christian Soize* \\ Université Paris-Est, Laboratoire Modélisation et Simulation Multi échelle, FRE3160 CNRS, 5 bd Descartes, \\ 77454 Marne-la-Vallée, France
}

Laurent Gagliardini

PSA Peugeot-Citroën, Acoustic division, 78943 Vélizy-Villacoublay, France

\begin{abstract}
The design of cars is mainly based on the use of computational models to analyze structural vibrations and internal acoustic levels. Considering the very high complexity of such structuralacoustic systems, and in order to improve the robustness of such computational structural-acoustic models, both model uncertainties and data uncertainties must to be taken into account. In this context, a probabilistic approach of uncertainties is implemented in an adapted computational structural-acoustic model. The two main problems are the experimental identification of the parameters controlling the uncertainty levels and the experimental validation. Relevant experiments have especially been developed for this research in order to constitute an experimental database devoted to structural vibrations and internal acoustic pressures. This database is used to perform the experimental identification of the probability model parameters and to validate the stochastic computational model.
\end{abstract}

\section{INTRODUCTION}

In the automotive industry, computational structuralacoustic models are nowadays intensively used to analyze the structural-acoustic behavior of vehicles in terms of structural vibrations and internal acoustic levels mainly for the low-frequency range. The present evolution is to extend such computational models to the mediumfrequency range. In this paper, we are interested in the booming noise consisting in studying the acoustic response at passengers' ears induced by engine structureborne excitations in the low-frequency band but also in the lower part of the medium-frequency band. Note that a very few papers have been published concerning the booming noise prediction in this frequency band using a computational model with or without experimental comparisons (Sol and Van-Herpe, 2001; Sung and Nefske, 2001; Hamdi et al., 2005; Hayashi et al., 2000). In addition, structural-acoustic analyses of cars in the highfrequency band are of a great importance for automotive engineering and can generally be treated by using statistical energy analysis and diffuse field methods for which numerous papers have been published in the last decade (see for instance Lyon and DeJong, 1995; Le Bot, 2002; Gagliardini et al., 2005; Shorter and Langley, 2005; Langley, 2007). Considering the very high complexity of such structural-acoustic systems, the mean computational structural-acoustic models do not allow sufficiently good predictions to be obtained and consequently, must be improved in implementing a model of uncertainties in order to increase the robustness of predictions. In this context, it is necessary to validate such computational

*Electronic address: christian.soize@univ-paris-est.fr structural-acoustic models with experiments. It should be noted that a very few complete and documented experimental databases are available in the literature. Only some elements concerning two databases can be found in the literature (Wood and Joachim, 1987 ; Kompella and Bernhard, 1996). Nevertheless, these two experimental databases cannot easily be used because there are no available computational structural-acoustic models associated with these databases. In order to get round this difficulty, a complete experimental campaign devoted to structural vibrations and internal acoustic pressures has been done for this research (Durand, 2007). This experimental database is presented in this paper and is used to perform an experimental identification and to validate the computational model. The problem related to such predictions with computational structural-acoustic models is extremely difficult due to the over-sensitiveness of structural dynamical responses with respect to manufacturing processes and due to small variabilities induced by the presence of optional extra around a main configuration. Note that automotive engineering requires to predict the structural-acoustic responses of a car of the same type with optional extra using only one computational structural-acoustic model. This over-sensitiveness may be seen by computation when applying design changes, but also experimentally when monitoring vehicle dispersions (Wood and Joachim, 1987; Kompella and Bernhard, 1996; Hills et al., 2004). Concerning the computational structural-acoustic models, many uncertainties are introduced by the mechanical-acoustical-mathematical modeling process due to the high complexity of the structure and of the internal acoustic cavity in terms of geometry, boundary conditions, material properties, etc. Even if a sophisticated structural-acoustic model is developed, model uncertainties and data uncertainties are inherent in such a computational structural-acoustic model. One 
objective of this paper is to use a representative computational structural-acoustic model developed by an automotive industry (Sol and Van Herpe, 2001; Durand et al., 2005a). In such a finite element model, (1) the structure is discretized with a reasonable fine scale, (2) the internal acoustic cavity is discretized with a coarse scale adapted to the frequency band of analysis and (3) the sound-proofing schemes located at the interface between the structure and the acoustic cavity is taken into account by a very simplified model. Clearly, the sound-proofing schemes could be discretized with a fine scale using the finite element method and formulations for porelastic materials (Attala et al., 2001; Hamdi et al., 2005). Such an approach has not been retained in this research. It seems that there is no paper available in the literature devoted to the development of a computational structuralacoustic model for cars including a model of uncertainties and associated with an experimental validation performed with a complete experimental database. In this paper, we present such a complete computational model including uncertainties modeling, experimental identification and experimental validation (Durand et al., 2004, 2005a and 2005b; Durand, 2007). It is known that several approaches can be used to take into account uncertainties in computational models of complex structural-acoustic systems for the low-frequency band (interval method, fuzzy sets approach, probabilistic approach, etc). In this paper, we have chosen to use the most efficient mathematical tool adapted to model uncertainties as soon as the probability theory can be used, i.e., the probabilistic approach. The mean computational structural-acoustic model is constructed from the designed system (conceptual system) using a mathematical-mechanical-acoustical modeling process. The mean computational model which is considered as a predictive model of the real system depends on parameters (or data). There are two types of uncertainties which are data uncertainties and model uncertainties. Data uncertainties are related to the parameters of the mean computational vibracoustic model and model uncertainties are induced by the modeling process. The "parametric probabilistic approach" is the most efficient and powerful method to address "data uncertainties" in predictive models (see for instance Schueller, 1997 and 2007, and Ghanem and Spanos, 2003 for stochastic finite element methods) as soon as the probability theory can be used but cannot address "model uncertainties". The "nonparametric probabilistic approach" recently proposed (Soize, 2000, 2001, 2003, 2005a and $2005 \mathrm{~b}$ ) is a way to address both model uncertainties and data uncertainties. The use of the parametric probabilistic approach of data uncertainties for structural-acoustic analysis of cars generally requires to introduce a very large number of random variables. This is due to the fact that the structural-acoustic responses are very sensitive to many parameters related to the geometry (such as plate and shell thicknesses, panels curvatures, etc.), to the spot welding points, to the boundary conditions, etc. Typically, several ten thousands parameters must be modeled by random variables. First, it should be noted that the construction of the probabilistic model of this large number of parameters is not so easy to carry out. The experimental identification of a very large number of probability distributions using measurements of the response of a structural-acoustic system (solving an inverse stochastic problem and consequently, solving an optimization problem) is completely unrealistic. In addition, as explained above, the parametric probabilistic approach does not allow model uncertainties to be taken into account and it is known that model uncertainties are significant in computational structural-acoustic models of cars. This is a reason why we propose to use the nonparametric probabilistic approach of uncertainties which allows data uncertainties but also model uncertainties to be taken into account. In addition, the nonparametric approach introduces a very small number of parameters (typically 7 parameters) which controls the level of uncertainties. In this condition, the experimental identification of these parameters is realistic and can be performed by solving the stochastic inverse problem using adapted mathematical-statistical tools. In this paper, we present such an approach. It should be noted that the nonparametric probabilistic approach of uncertainties has been used in the five last years for linear and nonlinear structural dynamical problems. Nevertheless, this approach has not yet been used for complex structural-acoustic systems and is presented in this paper.

In Section II, we present the experimental database which has specially been constructed for this research. Section III deals with the mean computational structuralacoustic model and Section IV is devoted to the stochastic reduced computational model. Finally, in Section V, we present the structural-acoustic response of the vehicle for which the experimental database has been carried out, the experimental identification of the probabilistic model of uncertainties and the experimental validation of the stochastic computational model.

\section{EXPERIMENTS}

Experiments which are described in this section have been performed in PSA Peugeot-Citroën facilities. The system under consideration is a given vehicle for which two sets of experiments have been defined. The first set is made up of structural vibration and structural-acoustic measurements. The second set is devoted to the acoustic measurements inside the internal acoustic cavity.

\section{A. Structural-vibration and structural-acoustic measurements}

The first set of experiments consists (1) of measurements of the structural Frequency Response Functions (FRFs) between one DOF for a given excitation force (vertical component of the force at one support of the engine) and normal accelerations to the structure at six 
given points and (2) of measurements of the structuralacoustic FRFs between nine DOFs of excitation corresponding to three given point forces at supports of the engine and the acoustic pressure at the driver ears located inside the internal acoustic cavity. For structuralvibration measurements, the excitation is performed by means of a hammer and the responses are identified with accelerometers. For the structural-acoustic measurements, the reciprocity method in acoustics is used. This means that the excitation is produced by an acoustic source located at the driver ears inside the internal acoustic cavity and the acceleration responses are measured at the nine DOFs introduced above. The frequency band of analysis is $[20,220] \mathrm{Hz}$. The experimental database has been constructed using 20 cars of the same type with different optional extras. The measurements have been realized at the exit of the assembly plant. Fig. 1 shows the car body, the structural driving point (vertical excitation) and the two structural observation points (denoted by Obs4 and Obs6) for which we present experimental results and for which comparisons with the computational structural-acoustic model is carried out. Fig. 2 shows the front of the car body and the three components of the three forces applied to the points shown on this figure and corresponding to the nine DOFs of excitation. Figs.

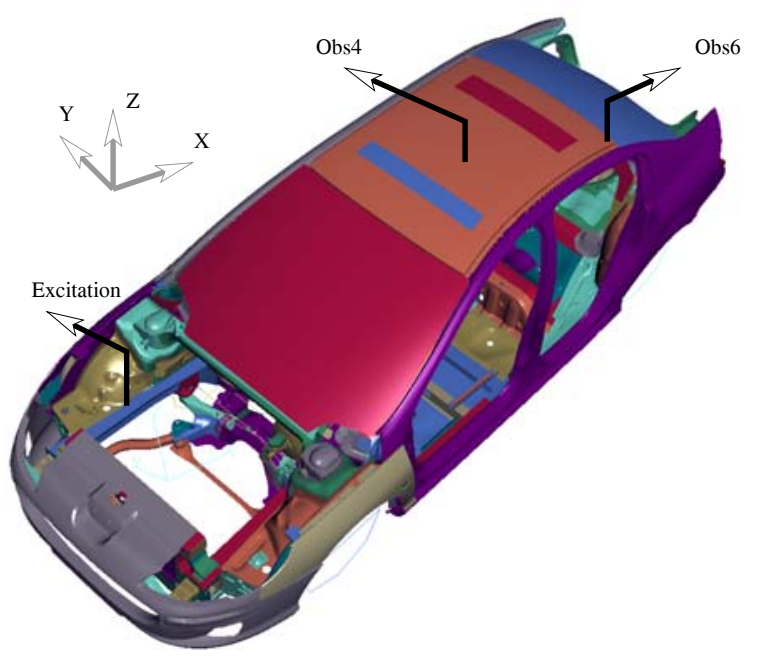

structural FRFs. Note that this technique is not used to perform the experimental identification of the structuralacoustic FRFs which are identified with the reciprocal acoustic method and for which the experimental identification is correct in the frequency band $[20,70] \mathrm{Hz}$. With the reciprocal acoustic method, we have not obtained any difficulties with respect to the coherence functions and then the measurements are validated in the frequency band $[20,220] \mathrm{Hz}$. The strategy used is then the following. The experimental structural FRFs is only used in the frequency band $[70,220] \mathrm{Hz}$ in order to identify the dispersion parameters of the nonparametric probabilistic model of uncertainties in the structure (and consequently, is not used in the frequency band $[20,70] \mathrm{Hz}$ ). The experimental structural-acoustic FRFs identified with the reciprocal acoustic method is used to validate the computational structural-acoustic model in all the frequency band $[20,220] \mathrm{Hz}$ including the frequency band [50, 183] $\mathrm{Hz}$ of the booming noise. Figs. 3 and 4 show the experimental dispersion on the structural responses obtained for the 20 cars of the same type. This experimental dispersion is due to the variability induced by the optional extras and induced by the manufacturing process. This dispersion is increasing with the frequency which is in coherence with the expected results. The experimental dispersion varies between 5 and $10 \mathrm{~dB}$ in the regions close to the resonances and varies from 15 to $30 \mathrm{~dB}$ in the neighborhood of the antiresonances. The experimental results concerning the structural-acoustic FRFs between the nine DOFs of the three given excitation forces and the acoustic pressure at the driver ears are not presented here in order to limit the number of figures. Nevertheless, these experimental results are directly used to construct 
the experimental booming noise synthesis which is presented below.

\section{B. Acoustic measurements inside the internal acoustic cavity}

The secpnd set of experiments consists of measure-

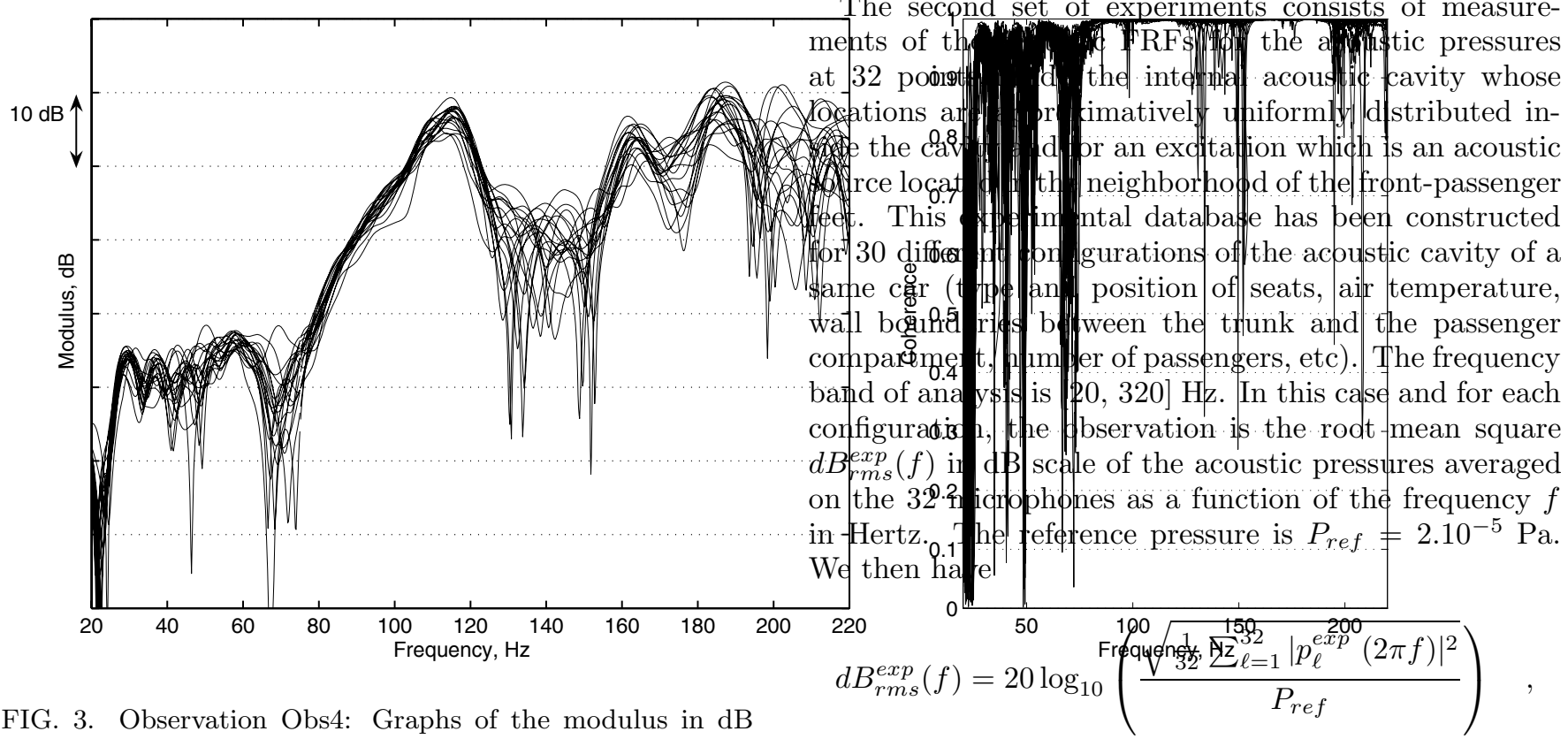

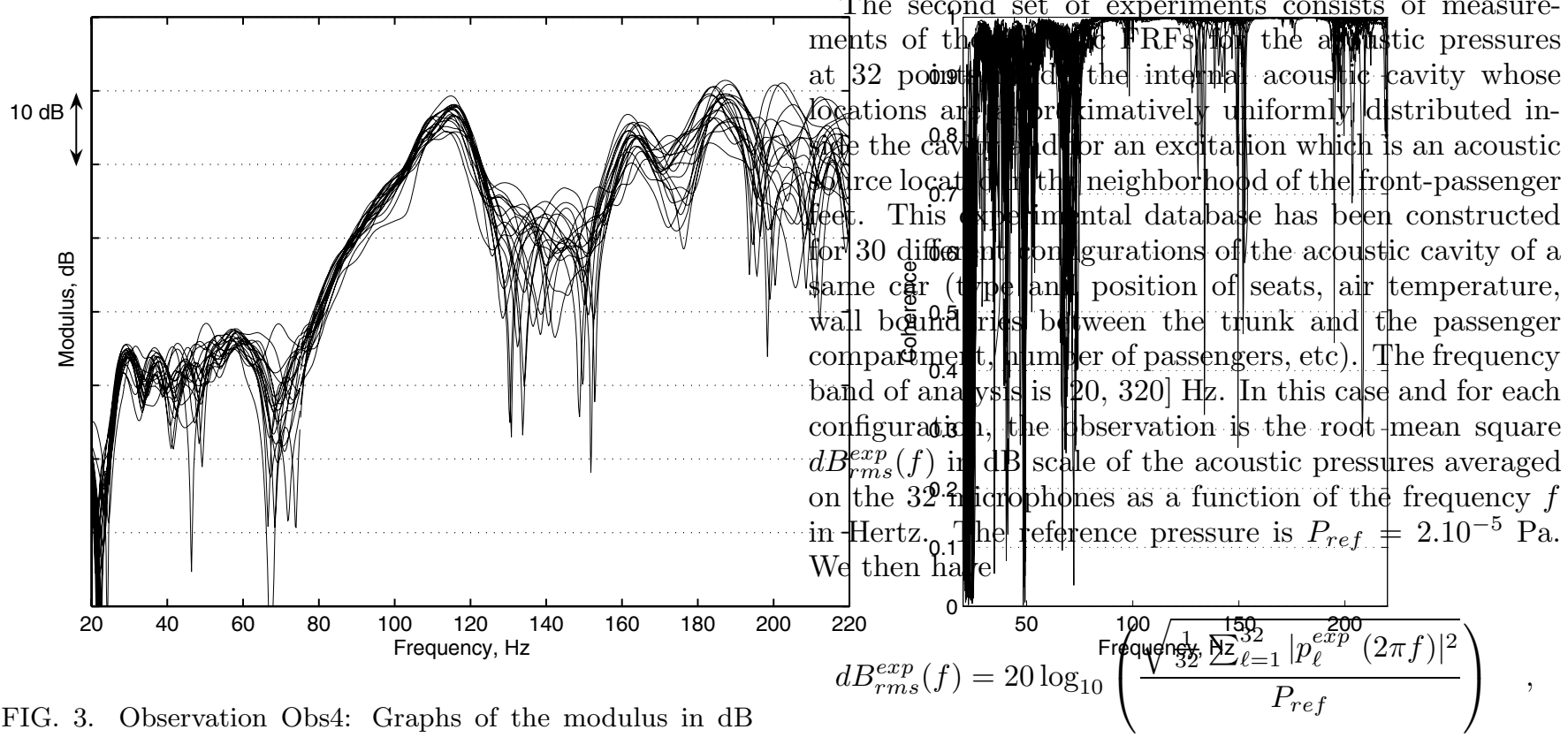

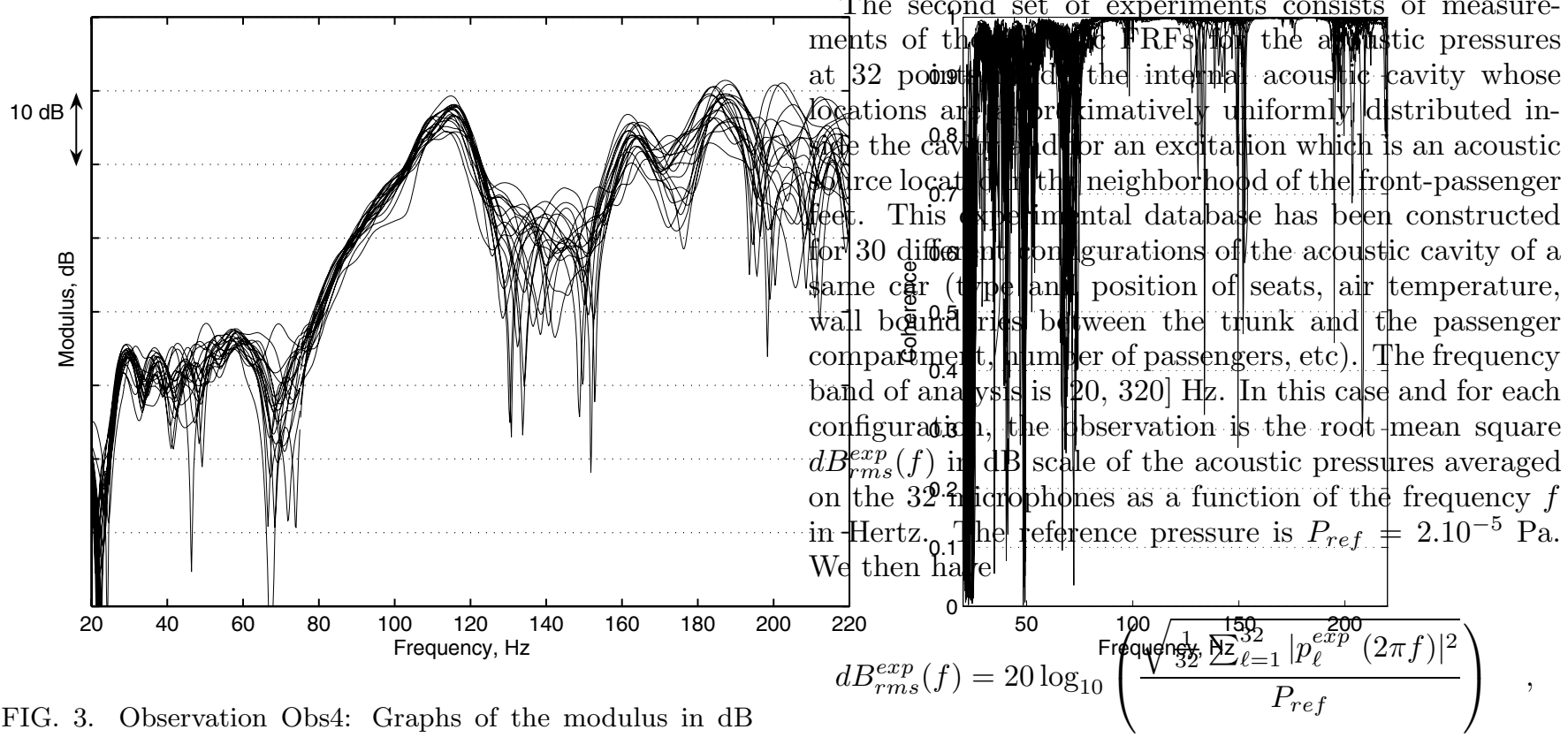
scale of the experimental structural FRFs as a function of the frequency in Hertz for the 20 cars (left figure). Graphs of the corresponding experimental coherence functions (right figure).

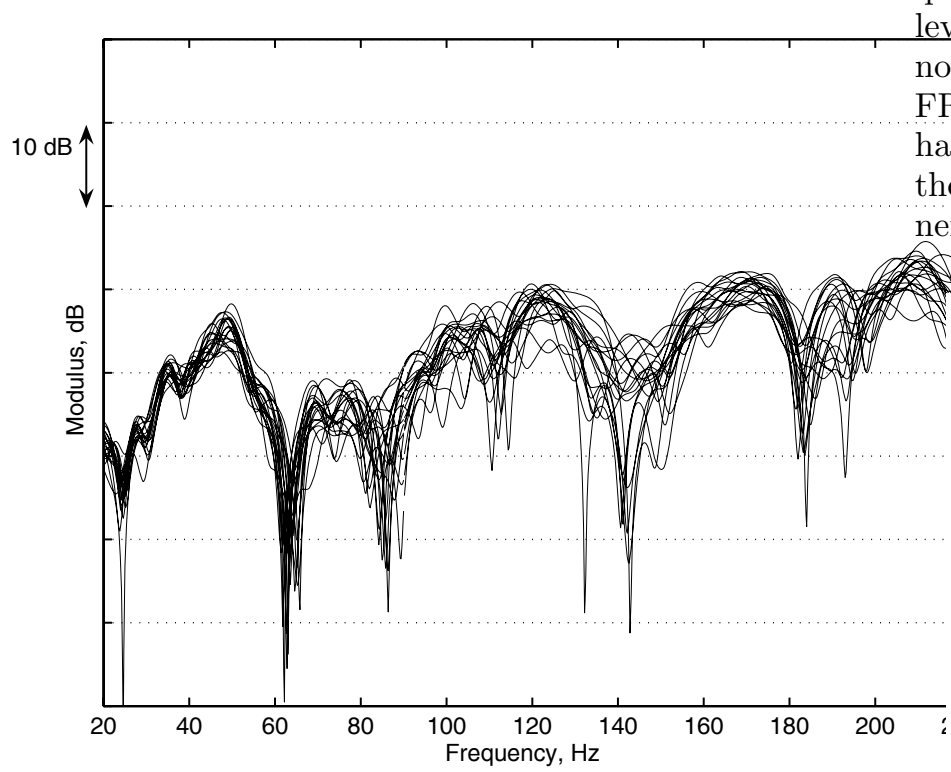

FIG. 4. Observation Obs6: Graphs of the modulus in $\mathrm{dB}$ scale of the experimental structural FRFs as a function of the frequency in Hertz for the 20 cars (left figure). Graphs of the corresponding experimental coherence functions (right figure). in which $p_{1}^{\exp }(\omega), \ldots, p_{32}^{\exp }(\omega)$ are the acoustic pressures measured by the 32 microphones at frequency $\omega$ in $\mathrm{rad} / \mathrm{s}$. Fig. 5 displays the graphs of $f \mapsto d B_{r m s}^{\exp }(f)$ for the 30 configurations of the internal acoustic cavity. This figure shows a low level of the experimental dispersion for this quantity with respect to the different configurations. The levels of dispersion vary from 2 to $3 \mathrm{~dB}$. It should be

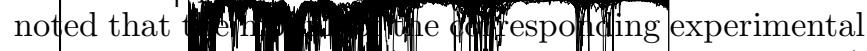

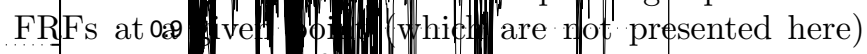

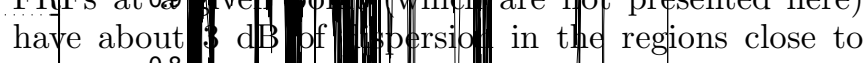
the resonan es and haf u p to $\beta$ dB of dispersion in the neighborhod

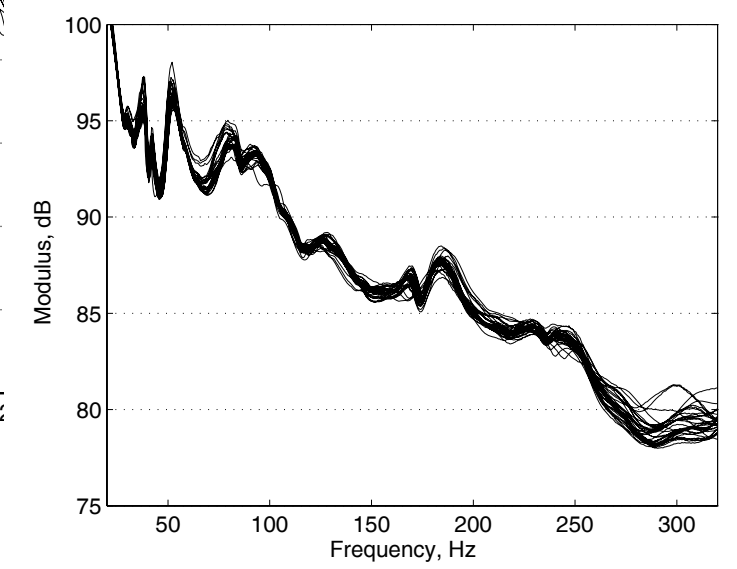

FIG. 5. For the 30 configurations of the internal acoustic cavity: graphs of $f \mapsto d B_{r m s}^{\exp }(f)$. 


\section{Experimental synthesis of booming noise}

As explained above, the measurements presented in Subsections A and B will be used to identify the probability model of uncertainties. Below we present the measurements which is used to validate the computational structural-acoustic model allowing the booming noise to be predicted in the frequency range $[50,183] \mathrm{Hz}$ corresponding to $[1500,5500] \mathrm{rpm}$ (rotation per minute of the engine). In this paper, the booming noise is defined as the modulus of the acoustic pressure in $\mathrm{dB}[\mathrm{A}]$ at the driver ears for structural excitation induced by the forces delivered by the engine at its 4 supports when the frequency of rotation varies in the frequency band of analysis. This function is denoted by $f \mapsto d B^{\exp }[A](f)$. Note that the forces delivered by the engine at its supports correspond to the second harmonic of rotation (for instance $1500 \mathrm{rpm}$ corresponds to a frequency equal to $50 \mathrm{~Hz}$ ). The scale used is the $\mathrm{dB}[\mathrm{A}]$ scale which corresponds to the weighting of the usual $\mathrm{dB}$ scale by the A-weighting. Fig. 6 displays the graph of $f \mapsto d B^{\exp }[A](f)$ related to the experimental synthesized booming noise and defined above. In this figure, it can be seen a very large experimental dispersion induced by the variability (optional extras) and by the manufacturing process. As for the experimental structural FRFs, the experimental dispersion varies between 5 and $10 \mathrm{~dB}$ in the regions close to the resonances and varies from 15 to $30 \mathrm{~dB}$ in the neighborhood of the antiresonances. These experimental results are in coherence with the experimental structural FRFs and can be explained by the fact that the experimental dispersion induced by uncertainties inside the internal acoustic cavity is relatively small with respect to structural uncertainties (see Fig. 5).

\section{REDUCED MEAN COMPUTATIONAL STRUCTURAL-ACOUSTIC MODEL}

The mean computational structural-acoustic model is developed in the context of 3D linear elastoacoustics, is formulated in the frequency domain and is discretized by using the finite element method. The structural-acoustic system is made up of a damped elastic structure coupled with a closed internal acoustic cavity filled with a dissipative acoustic fluid. The external acoustic fluid is air and its effects on the structural-acoustic system are assumed to be negligible in the low-frequency band of analysis which is considered in the paper and which is devoted to the booming noise prediction. The linear responses of the structural-acoustic system are studied around a static equilibrium state which is taken as the natural state at rest. The reduced mean computational structural-acoustic model is derived from the mean model using a modal analysis.

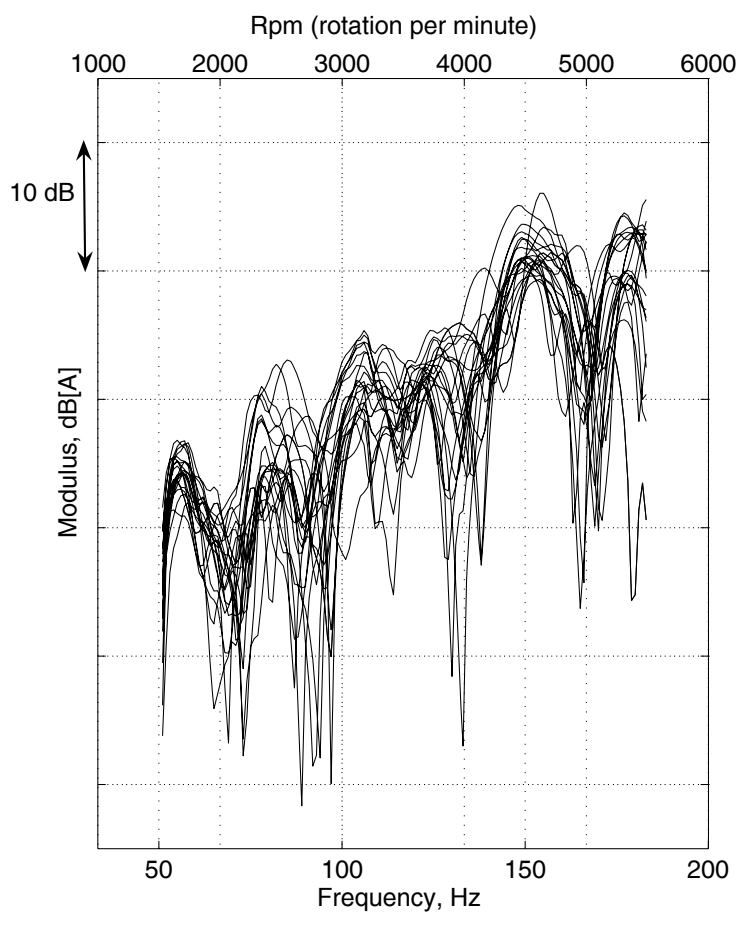

FIG. 6. Graph of $f \mapsto d B^{\exp }[A](f)$ related to the experimental synthesized booming noise.

\section{A. Description of the structural-acoustic system}

We then consider linear vibrations of a fixed damped structure $\Omega_{s}$ (the car body) subjected to external loads (engine excitations), coupled with its internal cavity $\Omega_{a}$ (passenger compartment and trunk) filled with a dissipative acoustic fluid (air). We are mainly interested in predicting the frequency responses of the structuralacoustic system in the frequency band of analysis $B=$ $\left[\omega_{\min }, \omega_{\max }\right] \mathrm{rad} / \mathrm{s}$ corresponding to $B_{f}=\left[f_{\min }, f_{\max }\right]$ Hz with $\omega_{\min }=2 \pi f_{\min }$ and $\omega_{\max }=2 \pi f_{\max }$. The physical space $\mathbb{R}^{3}$ is referred to a cartesian system and the generic point of $\mathbb{R}^{3}$ is denoted by $\boldsymbol{x}=\left(x_{1}, x_{2}, x_{3}\right)$. The system is defined in Fig. 7. Let $\partial \Omega_{s}=\Gamma_{s} \cup \Gamma_{0} \cup \Gamma_{a}$ be the boundary of $\Omega_{s}$. The outward unit normal to $\partial \Omega_{s}$ is denoted by $\boldsymbol{n}_{s}=\left(n_{s, 1}, n_{s, 2}, n_{s, 3}\right)$. The displacement field in $\Omega_{s}$ is denoted by $\boldsymbol{u}(\boldsymbol{x}, \omega)=\left(u_{1}(\boldsymbol{x}, \omega), u_{2}(\boldsymbol{x}, \omega), u_{3}(\boldsymbol{x}, \omega)\right)$. The structure is assumed to be fixed on the part $\Gamma_{0}$ of the boundary $\partial \Omega_{s}$. The internal acoustic cavity $\Omega_{a}$ is the bounded domain filled with a dissipative acoustic fluid. The boundary $\partial \Omega_{a}$ of $\Omega_{a}$ is $\Gamma$. The outward unit normal to $\partial \Omega_{a}$ is denoted by $\boldsymbol{n}_{a}=\left(n_{a, 1}, n_{a, 2}, n_{a, 3}\right)$ and we then have $\boldsymbol{n}_{a}=-\boldsymbol{n}_{s}$ on $\partial \Omega_{a}$. The pressure field in $\Omega_{a}$ is denoted by $p(\boldsymbol{x}, \omega)$. 


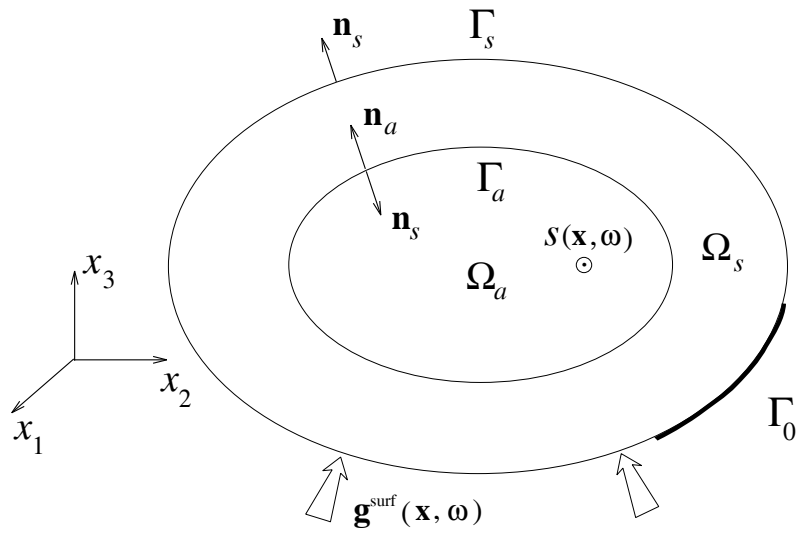

FIG. 7. Scheme of the structural-acoustic system.

\section{B. Mean boundary value problem for the structural-acoustic system}

The equation of the structural part is written (e.g., Trusdell, 1960; Ohayon and Soize, 1998) as

$$
-\omega^{2} \rho_{s} u_{i}-\frac{\partial \sigma_{i j}}{\partial x_{j}}=g_{i}^{v o l} \quad \text { in } \quad \Omega_{s}
$$

with the convention for summations over repeated Latin indices, in which $\rho_{s}$ is the mass density, $\sigma_{i j}$ is the stress tensor, $\boldsymbol{u}=\left(u_{1}, u_{2}, u_{3}\right)$ is the displacement field of the structure and $\boldsymbol{g}^{\text {vol }}=\left(g_{1}^{\text {vol }}, g_{2}^{\text {vol }}, g_{3}^{\text {vol }}\right)$ is the body force field applied to the structure. The boundary conditions are

$\sigma_{i j}(\boldsymbol{u}) n_{s, j}=g_{i}^{\text {surf }} \quad$ on $\quad \Gamma_{s} \quad, \quad \sigma_{i j}(\boldsymbol{u}) n_{s, j}=-p n_{s, i} \quad$ on

in which $\boldsymbol{g}^{\text {surf }}=\left(g_{1}^{\text {surf }}, g_{2}^{\text {surf }}, g_{3}^{\text {surf }}\right)$ is the surface force field applied to the structure. The damped structure is made up with a linear non homogeneous anisotropic viscoelastic material without memory. In the frequency domain, the constitutive equation is written as $\sigma_{i j}=$ $a_{i j k h} \epsilon_{k h}+i \omega b_{i j k h} \epsilon_{k h}$, in which the tensor $a_{i j k h}$ of the elastic coefficients and the tensor $b_{i j k h}$ of the damping coefficients of the material depend on $\boldsymbol{x}$, are independent of $\omega$ and have the usual properties of symmetry and positive definiteness. The strain tensor $\epsilon_{k h}$ is related to displacement field $\boldsymbol{u}$ by $\epsilon_{k h}=\left(\partial u_{k} / \partial x_{h}+\partial u_{h} / \partial x_{k}\right) / 2$. Concerning the internal dissipative acoustic fluid, we use the $\boldsymbol{u}-p$ formulation. The equation governing the vibration of the dissipative acoustic fluid occupying domain $\Omega_{a}$ is written as (Ohayon and Soize, 1998; Pierce, 1989; Lighthill, 1978)

$\frac{\omega^{2}}{\rho_{a} c_{a}^{2}} p+i \omega \frac{\tau}{\rho_{a}} \nabla^{2} p+\frac{1}{\rho_{a}} \nabla^{2} p=\frac{\tau}{\rho_{a}} c_{a}^{2} \nabla^{2} s-i \frac{\omega}{\rho_{a}} s$ in $\Omega_{a}$,

for which the boundary conditions are

$$
\frac{1}{\rho_{a}}(1+i \omega \tau) \frac{\partial p}{\partial \boldsymbol{n}_{a}}=\omega^{2} \boldsymbol{u} \cdot \boldsymbol{n}_{a}+\tau \frac{c_{a}^{2}}{\rho_{a}} \frac{\partial s}{\partial \boldsymbol{n}_{a}} \quad \text { on } \quad \Gamma_{a},
$$

where $\rho_{a}$ is the mass density of the acoustic fluid at equilibrium, $c_{a}$ is the speed of sound, $\tau$ is the coefficient due to the viscosity of the fluid (the coefficient due to thermal conduction is neglected) and where $s(\boldsymbol{x}, \omega)$ is the acoustic source density.

\section{Mean computational structural-acoustic model}

The finite element method (Zienkiewicz and Taylor, 2000) is used to solve numerically the above boundary value problem. We consider a finite element mesh of the structure $\Omega_{s}$ and of the internal acoustic fluid $\Omega_{a}$. Let $\underline{\boldsymbol{u}}^{s}=\left(\underline{u}_{1}^{s}, \ldots, \underline{u}_{n}^{s}\right)$ be the complex vector of the $n_{s}$ degrees of freedom (DOFs) of the structure according to the finite element discretization of the displacement field $\boldsymbol{u}$. Let $\underline{\boldsymbol{p}}^{a}=\left(\underline{p}_{1}^{a}, \ldots, \underline{p}_{n_{a}}^{a}\right)$ be the complex vector of the $n_{a}$ DOFs of the acoustic fluid to the finite element discretization of the pressure field $p$. The finite element discretization of the boundary value problem in terms of $\boldsymbol{u}$ and $p$ (Ohayon and Soize, 1998), defined by Eqs.(1) to (4) with the Dirichlet condition on $\Gamma_{0}$ yields the mean computational structural-acoustic model,

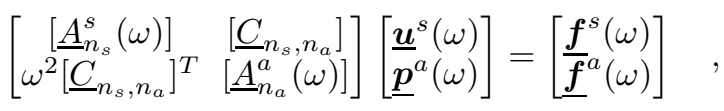

where $\left[\underline{A}_{n_{s}}^{s}(\omega)\right]$ is the dynamical stiffness matrix of the damped structure in vacuo which is a symmetric $\left(n_{s} \times n_{s}\right)$ complex matrix such that

$$
\left[\underline{A}_{n_{s}}^{s}(\omega)\right]=-\omega^{2}\left[\underline{M}_{n_{s}}^{s}\right]+i \omega\left[\underline{D}_{n_{s}}^{s}\right]+\left[\underline{K}_{n_{s}}^{s}\right],
$$

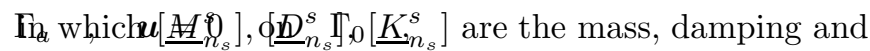
stiffness matrices of the structure which are positivedefinite symmetric $\left(n_{s} \times n_{s}\right)$ real matrices. In Eq.(5), $\left[\underline{A}_{n_{a}}^{a}(\omega)\right]$ is the dynamical stiffness matrix of the dissipative acoustic fluid which is a symmetric $\left(n_{a} \times n_{a}\right)$ complex matrix such that

$$
\left[\underline{A}_{n_{a}}^{a}(\omega)\right]=-\omega^{2}\left[\underline{M}_{n_{a}}^{a}\right]+i \omega\left[\underline{D}_{n_{a}}^{a}\right]+\left[\underline{K}_{n_{a}}^{a}\right],
$$

in which $\left[\underline{M}_{n_{a}}^{a}\right],\left[\underline{D}_{n_{a}}^{a}\right],\left[\underline{K}_{n_{a}}^{a}\right]$ are the "mass", "damping" and "stiffness" matrices of the acoustic cavity with fixed coupling interface. The matrix $\left[\underline{M}_{n}^{a}\right]$ is a positivedefinite symmetric $\left(n_{a} \times n_{a}\right)$ real matrix, $\left[\underline{D}_{n_{a}}^{a}\right]$ and $\left[\underline{K}_{n_{a}}^{a}\right]$ are positive-semidefinite symmetric $\left(n_{a} \times n_{a}\right)$ real matrices whose ranks are $n_{a}-1$. The matrix $\left[C_{n_{s}, n_{a}}\right]$ is the structural-acoustic coupling matrix which is a $\left(n_{s} \times n_{a}\right)$ real matrix.

\section{Reduced mean computational structural-acoustic model}

The projection of the mean computational structuralacoustic model on the structural modes in vacuo and on the acoustic modes of the acoustic cavity with fixed coupling interface yields the reduced mean computational structural-acoustic model. The structural modes 
in vacuo and the acoustic modes of the cavity with fixed coupling interface are calculated by solving the two generalized eigenvalue problems

$$
\begin{gathered}
{\left[\underline{K}_{n_{s}}^{s}\right] \underline{\boldsymbol{\psi}}=\underline{\lambda}^{s}\left[\underline{M}_{n_{s}}^{s}\right] \underline{\boldsymbol{\psi}}} \\
{\left[\underline{K}_{n_{a}}^{a}\right] \underline{\boldsymbol{\phi}}=\underline{\lambda}^{a}\left[\underline{M}_{n_{a}}^{a}\right] \underline{\boldsymbol{\phi}} .}
\end{gathered}
$$

The eigenvectors verify the usual orthogonal properties (Bathe and Wilson, 1976; Géradin and Rixen, 1994; Ohayon and Soize; 1998). The structural displacement is written as

$$
\underline{\boldsymbol{u}}^{s}(\omega)=[\underline{\Psi}] \underline{\boldsymbol{q}}^{s}(\omega)
$$

in which $[\underline{\Psi}]$ is the $\left(n_{s} \times n\right)$ real matrix whose columns are constituted of the $n$ structural modes associated with the $n$ first positive eigenvalues (the $n$ first structural eigenfrequencies). The internal acoustic cavity has one constant pressure mode and $m-1$ acoustic modes. The internal acoustic pressure is written as

$$
\underline{\boldsymbol{p}}^{a}(\omega)=[\underline{\Phi}] \underline{\boldsymbol{q}}^{a}(\omega)
$$

in which $[\underline{\Phi}]$ is the $\left(n_{a} \times m\right)$ real matrix whose columns are constituted (1) of the constant pressure mode associated with the zero eigenvalue and (2) of the acoustic modes associated with the positive eigenvalues (the $m-1$ first acoustical eigenfrequencies). It should be noted that the constant pressure mode is kept in order to model the quasi-static variation of the internal fluid pressure induced by the deformation of the coupling interface (Ohayon and Soize, 1998). Using Eqs. (8) and (9), the projection of Eq. (5) yields the reduced mean matrix model of the structural-acoustic system

$$
\left[\begin{array}{cc}
{\left[\underline{\mathcal{A}}_{n}^{s}(\omega)\right]} & {\left[\underline{\mathcal{C}}_{n, m}\right]} \\
\omega^{2}\left[\underline{\mathcal{C}}_{n, m}\right]^{T} & {\left[\underline{\mathcal{A}}_{m}^{a}(\omega)\right]}
\end{array}\right]\left[\begin{array}{l}
\underline{\boldsymbol{q}}^{s}(\omega) \\
\underline{\underline{\boldsymbol{q}}}^{a}(\omega)
\end{array}\right]=\left[\begin{array}{l}
\underline{\mathcal{F}}^{s}(\omega) \\
\underline{\mathcal{F}}^{a}(\omega)
\end{array}\right],
$$

where $\left[\underline{\mathcal{C}}_{n, m}\right]=[\underline{\Psi}]^{T}\left[\underline{C}_{n_{s}, n_{a}}\right][\underline{\Phi}],\left[\underline{\mathcal{F}}^{s}(\omega)\right]=[\underline{\Psi}]^{T}\left[\underline{\boldsymbol{f}}^{s}(\omega)\right]$ and $\left[\underline{\mathcal{F}}^{a}(\omega)\right]=[\underline{\Phi}]^{T}\left[\underline{\boldsymbol{f}}^{a}(\omega)\right]$. The generalized dynamical stiffness matrix $\left[\underline{\mathcal{A}}_{n}^{s}(\bar{\omega})\right]$ of the damped structure is written as

$$
\left[\underline{\mathcal{A}}_{n}^{s}(\omega)\right]=-\omega^{2}\left[\underline{\mathcal{M}}_{n}^{s}\right]+i \omega\left[\underline{\mathcal{D}}_{n}^{s}\right]+\left[\underline{\mathcal{K}}_{n}^{s}\right],
$$

in which $\left[\underline{\mathcal{M}}_{n}^{s}\right]=[\underline{\Psi}]^{T}\left[\underline{M}_{n s}^{s}\right][\underline{\Psi}]$ and $\left[\underline{\mathcal{K}}_{n}^{s}\right]=[\underline{\Psi}]^{T}\left[\underline{K}_{n_{s}}^{s}\right][\underline{\Psi}]$ are positive-definite diagonal $(n \times n)$ real matrices and where $\left[\underline{\mathcal{D}}_{n}^{s}\right]=[\underline{\Psi}]^{T}\left[\underline{D}_{n}^{s}\right][\underline{\Psi}]$ is, in general, a positivedefinite full $(n \times n)$ real matrix. The generalized dynamical stiffness matrix $\left[\underline{\mathcal{A}}_{m}^{a}(\omega)\right]$ of the dissipative acoustic fluid is written as

$$
\left[\underline{\mathcal{A}}_{m}^{a}(\omega)\right]=-\omega^{2}\left[\underline{\mathcal{M}}_{m}^{a}\right]+i \omega\left[\underline{\mathcal{D}}_{m}^{a}\right]+\left[\underline{\mathcal{K}}_{m}^{a}\right],
$$

in which $\left[\underline{\mathcal{M}}_{m}^{a}\right]=[\underline{\Phi}]^{T}\left[\underline{M}_{n_{a}}^{a}\right][\underline{\Phi}]$ is a positive-definite diagonal $(m \times m)$ real matrix and where $\left[\underline{\mathcal{D}}_{m}^{a}\right]=$ $[\underline{\Phi}]^{T}\left[\underline{D}_{n_{a}}^{a}\right][\underline{\Phi}]$ and $\left[\underline{\mathcal{K}}_{m}^{a}\right]=\left[\underline{\Phi}^{T}\left[\underline{K}_{n_{a}}^{a}\right][\underline{\Phi}]\right.$ are positivesemidefinite diagonal $(m \times m)$ real matrices of rank $m-1$.

\section{STOCHASTIC REDUCED COMPUTATIONAL STRUCTURAL-ACOUSTIC MODEL}

As explained in the Introduction, both data uncertainties and model uncertainties can be taken into account by using the nonparametric probabilistic approach of uncertainties. Such an approach (Soize, 2000, 2001 and 2005a) has been used in linear and non linear structural dynamics. Nevertheless, this approach has not yet been used for complex structural-acoustic systems which requires the use of extended results concerning random matrix theory in order to take into account model uncertainties for the structural-acoustic coupling operator. Such extended results have been recently proposed by (Soize, 2005b).

\section{A. Constructing the stochastic model}

The use of the nonparametric probabilistic approach (Durand, 2007) of both model uncertainties and data uncertainties for the structure, the acoustic cavity and the structural-acoustic coupling consists (1) in modeling the generalized mass $\left[\underline{\mathcal{M}}_{n}^{s}\right]$, damping $\left[\underline{\mathcal{D}}_{n}^{s}\right]$ and stiffness $\left[\underline{\mathcal{K}}_{n}^{s}\right]$ matrices of the structure by random matrices $\left[\boldsymbol{M}_{n}^{s}\right],\left[\overline{\boldsymbol{D}}_{n}^{s}\right]$ and $\left[\boldsymbol{K}_{n}^{s}\right]$ whose dispersion parameters are denoted by $\delta_{M^{s}}, \delta_{D^{s}}$ and $\delta_{K^{s}}$ respectively; (2) in modeling the generalized mass $\left[\underline{\mathcal{M}}_{m}^{a}\right]$, damping $\left[\underline{\mathcal{D}}_{m}^{a}\right]$ and stiffness $\left[\underline{\mathcal{K}}_{m}^{a}\right]$ matrices of the acoustic cavity by random matrices $\left[\boldsymbol{M}_{m}^{a}\right]$, $\left[\boldsymbol{D}_{m}^{a}\right]$ and $\left[\boldsymbol{K}_{m}^{a}\right]$ whose dispersion parameters are denoted by $\delta_{M^{a}}, \delta_{D^{a}}$ and $\delta_{K^{a}}$ respectively; (3) in modeling the generalized structural-acoustic coupling matrix $\left[\underline{\mathcal{C}}_{n, m}\right]$ by a random matrix $\left[\boldsymbol{C}_{n, m}\right]$ whose dispersion parameter is denoted by $\delta_{C}$. The explicit construction of the probability distribution of these random matrices is given by (Soize 2000, 2001 and 2005b; Durand, 2007) for random matrices $\left[\boldsymbol{M}_{n}^{s}\right],\left[\boldsymbol{D}_{n}^{s}\right],\left[\boldsymbol{K}_{n}^{s}\right],\left[\boldsymbol{M}_{m}^{a}\right],\left[\boldsymbol{D}_{m}^{a}\right],\left[\boldsymbol{K}_{m}^{a}\right]$ and $[\boldsymbol{C}]$. Let $[\boldsymbol{H}]$ be anyone of these random matrices. The probability distribution of such a random matrix $[\boldsymbol{H}]$ depends on its mean value $[\underline{H}]=E\{[\boldsymbol{H}]\}$ where $E$ is the mathematical expectation and depends on its dispersion parameter $\delta_{H}$ which must be taken independent of the matrix dimension. An algebraic representation of random matrix $[\boldsymbol{H}]$ has been developed and allows independent realizations to be constructed for a stochastic solver based on the Monte Carlo numerical simulation. This algebraic representation is recalled below. For random matrices $\left[\boldsymbol{M}_{n}^{s}\right],\left[\boldsymbol{D}_{n}^{s}\right],\left[\boldsymbol{K}_{n}^{s}\right],\left[\boldsymbol{M}_{m}^{a}\right],\left[\boldsymbol{D}_{m}^{a}\right]$ and $\left[\boldsymbol{K}_{m}^{a}\right]$, random matrix $[\boldsymbol{H}]$ is then a symmetric positive-definite (or positivesemidefinite) real-valued random matrix and $[\boldsymbol{H}]$ is written as $[\boldsymbol{H}]=\left[\underline{L}_{H}\right]^{T}\left[\boldsymbol{G}_{H}\right]\left[\underline{L}_{H}\right]$ in which $[\underline{H}]=\left[\underline{L}_{H}\right]^{T}\left[\underline{L}_{H}\right]$ and where $\left[\boldsymbol{G}_{H}\right]$ is the random matrix germ. Note that if $[\underline{H}]$ is positive-semidefinite than the factorization is not a Cholesky factorization and is obtained by another algebraic algorithm. When $[\boldsymbol{H}]$ is the rectangular matrix $\left[\boldsymbol{C}_{n, m}\right]$ (see Soize 2005b), using the following polar decomposition $\left[\underline{\mathcal{C}}_{n, m}\right]=[\underline{U}][\underline{T}]$ with $[\underline{U}]^{T}[\underline{U}]=[I]$ where $[\underline{T}]$ is a positive-definite matrix which can then be factorized as $[\underline{T}]=\left[\underline{L}_{C}\right]^{T}\left[\underline{L}_{C}\right]$, the random matrix $\left[\boldsymbol{C}_{n, m}\right]$ can 
then be written as $\left[\boldsymbol{C}_{n, m}\right]=[\underline{U}]\left[\underline{L}_{C}\right]^{T}\left[\boldsymbol{G}_{H}\right]\left[\underline{L}_{C}\right]$ in which $\left[\boldsymbol{G}_{H}\right]$ is another random matrix germ. The stochastic reduced model of the uncertain structural-acoustic system for which the reduced mean model is defined by Eqs. (8) to (10) is written, for all $\omega$ fixed in the frequency band of analysis as

$$
\boldsymbol{U}^{s}(\omega)=[\underline{\Psi}] \boldsymbol{Q}^{s}(\omega) \quad, \quad \boldsymbol{P}^{a}(\omega)=[\underline{\Phi}] \boldsymbol{Q}^{a}(\omega),
$$

in which the $\mathbb{C}^{n}$-valued random variable $\boldsymbol{Q}^{s}(\omega)=$ $\left(Q_{1}^{s}(\omega), \ldots, Q_{n}^{s}(\omega)\right)$ and the $\mathbb{C}^{m}$-valued random variable $\boldsymbol{Q}^{a}(\omega)=\left(Q_{1}^{a}(\omega), \ldots, Q_{m}^{a}(\omega)\right)$ are the solution of the following random matrix equation

$$
\left[\begin{array}{cc}
{\left[\mathbf{A}_{n}^{s}(\omega)\right]} & {\left[\mathbf{C}_{n, m}\right]} \\
\omega^{2}\left[\mathbf{C}_{n, m}\right]^{T} & {\left[\mathbf{A}_{m}^{a}(\omega)\right]}
\end{array}\right]\left[\begin{array}{l}
\boldsymbol{Q}^{s}(\omega) \\
\boldsymbol{Q}^{a}(\omega)
\end{array}\right]=\left[\begin{array}{l}
\mathcal{F}^{s}(\omega) \\
\underline{\mathcal{F}}^{a}(\omega)
\end{array}\right],
$$

in which the random complex matrices $\left[\boldsymbol{A}_{n}^{s}(\omega)\right]$ and $\left[\boldsymbol{A}_{m}^{a}(\omega)\right]$ are defined by $\left[\boldsymbol{A}_{n}^{s}(\omega)\right]=-\omega^{2}\left[\boldsymbol{M}_{n}^{s}\right]+i \omega\left[\boldsymbol{D}_{n}^{s}\right]+$ $\left[\boldsymbol{K}_{n}^{s}\right]$ and where $\left[\boldsymbol{A}_{m}^{a}(\omega)\right]=-\omega^{2}\left[\boldsymbol{M}_{m}^{a}\right]+i \omega\left[\boldsymbol{D}_{m}^{a}\right]+\left[\boldsymbol{K}_{m}^{a}\right]$. Let $\boldsymbol{G}_{H}$ be the positive-definite symmetric $(\nu \times \nu)$ random matrix representing $\left[\boldsymbol{G}_{M_{n}^{s}}\right],\left[\boldsymbol{G}_{D_{n}^{s}}\right],\left[\boldsymbol{G}_{K_{n}^{s}}\right],\left[\boldsymbol{G}_{M_{m}^{a}}\right]$, $\left[\boldsymbol{G}_{D_{m}^{a}}\right],\left[\boldsymbol{G}_{K_{m}^{a}}\right]$ or $\left[\boldsymbol{G}_{C_{n, m}}\right]$. The following algebraic representation of random matrix $\left[\boldsymbol{G}_{H}\right]$ allows independent realizations of $\left[\boldsymbol{G}_{H}\right]$ to be constructed. Random matrix $\left[\boldsymbol{G}_{H}\right]$ is written $\left[\boldsymbol{G}_{H}\right]=\left[\boldsymbol{L}_{H}\right]^{T}\left[\boldsymbol{L}_{H}\right]$ in which $\left[\boldsymbol{L}_{H}\right]$ is a random upper triangular $(\nu \times \nu)$ real matrix whose random elements are independent random variables defined as follows:

(1) For $j<j^{\prime}$, the real-valued random variable $\left[\boldsymbol{L}_{H}\right]_{j j^{\prime}}$ is written as $\left[\boldsymbol{L}_{H}\right]_{j j^{\prime}}=\sigma_{\nu} U_{j j^{\prime}}$ in which $\sigma_{\nu}=\delta_{H}(\nu+1)^{-1 / 2}$ and where $U_{j j^{\prime}}$ is a real-valued Gaussian random variable with zero mean and variance equal to 1 . The parameter $\delta_{H}$ controlling the dispersion level of random matrix $[\boldsymbol{H}]$ is such that $\delta_{H}=\sqrt{E\left\{\left\|\left[\boldsymbol{G}_{H}\right]-[I]\right\|_{F}^{2}\right\} / \nu}$ in which $[I]$ is the identity matrix and where the subindex $F$ corresponds to the Frobenius norm.

(2) For $j=j^{\prime}$, the positive-valued random variable $\left[\boldsymbol{L}_{H}\right]_{j j^{\prime}}$ is written as $\left[\boldsymbol{L}_{H}\right]_{j j^{\prime}}=\sigma_{\nu} \sqrt{2 V_{j}}$ in which $\sigma_{\nu}$ is defined above and where $V_{j}$ is a positive-valued gamma random variable whose probability density function $p_{V_{j}}$ with respect to $d v$ is written as

$$
p_{V_{j}}(v)=\mathbb{1}_{\mathbb{R}^{+}}(v) \frac{1}{\Gamma\left(\frac{\nu+1}{2 \delta_{H}^{2}}+\frac{1-j}{2}\right)} v^{\left(\frac{\nu+1}{2 \delta_{H}^{2}}-\frac{1-j}{2}\right)} e^{-v} .
$$

\section{B. Confidence region of the random responses}

Let $\omega \mapsto W(\omega)$ from $B$ into $\mathbb{R}$ be a random observation of the structural-acoustic system in the frequency domain for which $n_{r}$ independent realizations $\omega \mapsto W\left(\omega, \theta_{1}\right), \ldots, \omega \mapsto W\left(\omega, \theta_{n_{r}}\right)$ are computed using the stochastic model presented in Subsection IV.A with the Monte Carlo numerical simulation. The confidence region associated with the probability level $\alpha$ for the random function $\{W(\omega), \omega \in B\}$ is constructed by using the method of quantiles (Serfling, 1980). For fixed $\omega$ in $B$, let $F_{W(\omega)}$ be the cumulative distribution function (continuous from the right) of random variable $W(\omega)$ which is such that $F_{W(\omega)}(w)=P(W(\omega) \leq w)$. For $0<p<1$, the $p$-th quantile or fractile of $F_{W(\omega)}$ is defined as $\zeta(p)=\inf \left\{w: F_{W(\omega)}(w) \geq p\right\}$. Then the upper envelope $w^{+}(\omega)$ and the lower envelope $w^{-}(\omega)$ of the confidence region are defined by $w^{+}(\omega)=\zeta((1+\alpha) / 2)$ and $w^{-}(\omega)=\zeta((1-\alpha) / 2)$. The estimation of $w^{+}(\omega)$ and $w^{-}(\omega)$ is performed by using the sample quantiles.

\section{STRUCTURAL-ACOUSTIC RESPONSE OF THE VEHICLE}

In this section, we present the experimental identification of the dispersion parameters of the nonparametric probabilistic approach of uncertainties for vibroacoustic analysis of the vehicle for which the experimental database has been presented in Section II. We then present the validation of the structural-acoustic model including uncertainties in comparing the identified stochatic computational model with experiments (Durand, 2007).

\section{A. Description of the mean computational structural-acoustic model and experimental comparisons}

The mean computational structural-acoustic model of the vehicle is a finite element model with 978,733 structural dofs and 8,139 acoustic pressure dofs in the internal acoustic cavity. Fig. 8 displays the finite element mesh of the structure and Fig. 9 shows the finite element mesh of the acoustic cavity. Finally, Fig. 10 deals with the finite element mesh of the computational structuralacoustic model. The reduced mean computational model is constructed using $n=1,723$ elastic modes of the structure in vacuo and $m=57$ acoustic modes of the internal acoustic cavity with rigid walls. These values of $n$ and $m$ have been deduced from a mean-square convergence analysis of the random response. Concerning the damping of the mean computational structural-acoustics model, the generalized damping matrix of the structure and of the internal acoustic cavity are assumed to be diagonal matrices for all frequencies in $B$. Note that such a diagonal assumption introduces model uncertainties in the mean computational model which are taken into account by the nonparametric probabilistic approach. Consequently, the damping part in the mean model is described in terms of damping rates which have been fixed to their nominal values. As it can be seen on the experimental frequency response functions, the structural damping is significant and the internal acoustic cavity damping is relatively high due to the presence of absorbing materials. For the structural FRFs, Figs. 11 and 12 display the comparisons between the mean computational structural-acoustic model predictions and the experiments for the moduli of the FRFs in $\mathrm{dB}$ concerning observations Obs4 (Fig. 11) and Obs6 (Fig. 12) and 


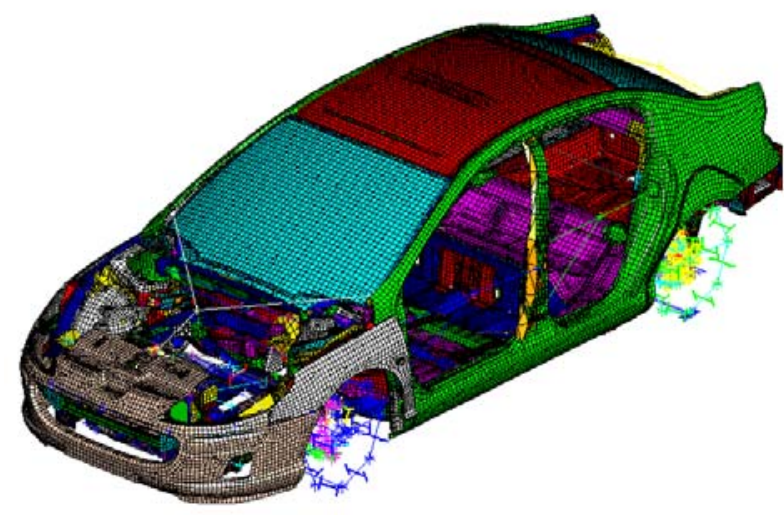

FIG. 8. Finite element mesh of the structure: 978,733 structural dofs.

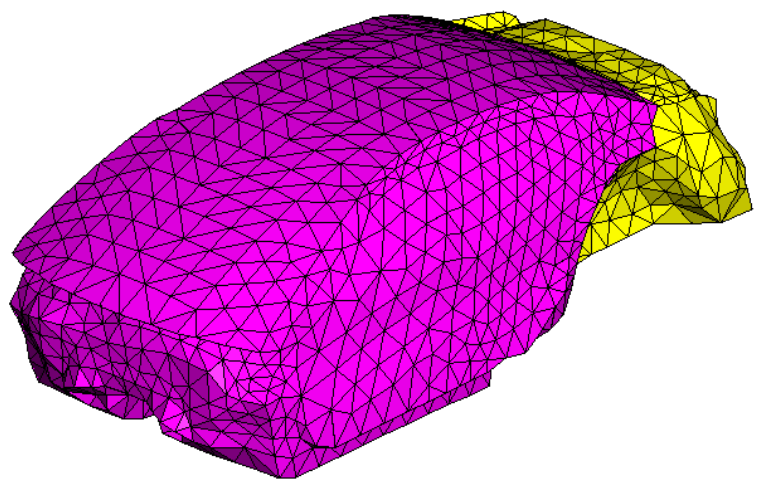

FIG. 9. Finite element mesh of the acoustic cavity: 8,139 acoustic pressure dofs.

for the 20 cars. For the root mean square of the acoustic pressures averaged on the 32 microphones inside the internal acoustic cavity (see Subsection II.B), Fig. 13 displays the graphs of $f \mapsto d B_{r m s}^{e x p}(f)$ for the 30 configurations of the internal acoustic cavity and the corresponding graph $f \mapsto d B_{r m s}(f)$ calculated with the mean computational model. Fig. 14 displays the graph of $f \mapsto d B^{\exp }[A](f)$ related to the experimental synthesized booming noise (see Subsection II.C) and the corresponding graph $f \mapsto d B[A](f)$ calculated with the mean computational model. Figs. 11 to 14 show that there are significant differences between measurements and numerical simulations which are not due to the variabilities of experiments (optional extras and manufacturing process) but are mainly due to model uncertainties. As explained in Section I, these figures clearly show that both data uncertainties and model uncertainties have to be taken into account in the mean computational structural-acoustic model in order to improve the predictability and the ro-

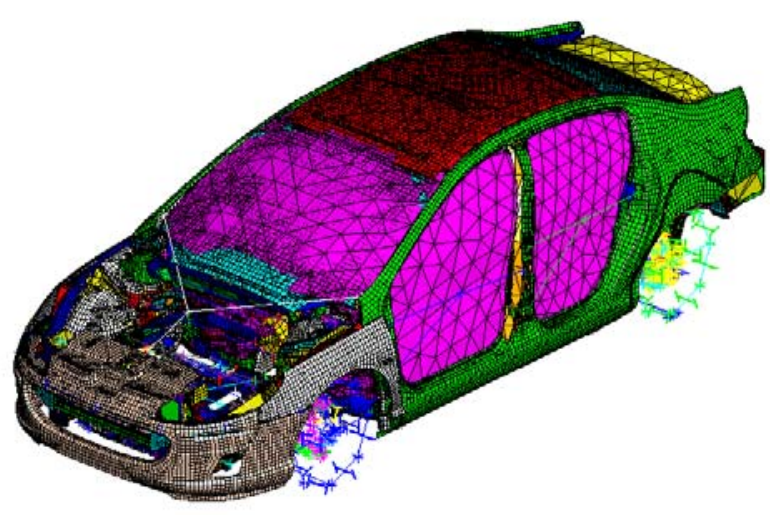

FIG. 10. Finite element mesh of the computational structural-acoustic model.

bustness of the predictions.

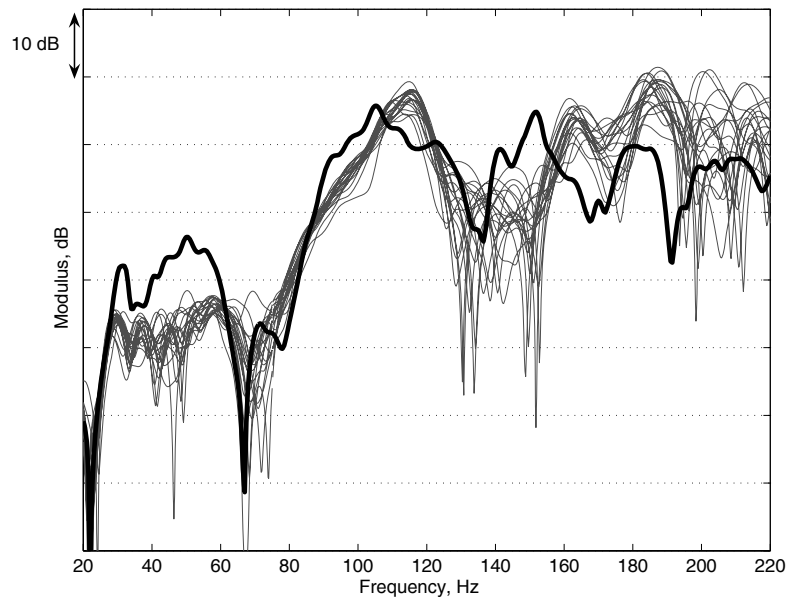

FIG. 11. Comparisons of the mean computational model results with the experiments. Graphs of the modulus in dB scale of the structural FRF for observation Obs4: experiments for the 20 cars (grey lines), mean computational model (thick solid line).

\section{B. Methodology and assumptions for the experimental identification of the dispersion parameters}

The methodology and the assumptions used to identify the dispersion parameters $\delta_{M^{s}}, \delta_{D^{s}}, \delta_{K^{s}}$ for the structure, $\delta_{M^{a}}, \delta_{D^{a}}, \delta_{K^{a}}$ for the internal acoustic cavity and $\delta_{C}$ for the structural-acoustic coupling interface are the following.

(1) The dispersion parameters $\delta_{M^{a}}, \delta_{D^{a}}$ and $\delta_{K^{a}}$ of the internal acoustic cavity are identified using the experimental database defined in Subsection II.B. For this identification it is assumed that $\delta_{M^{a}}=\delta_{D^{a}}=\delta_{K^{a}}$. First 


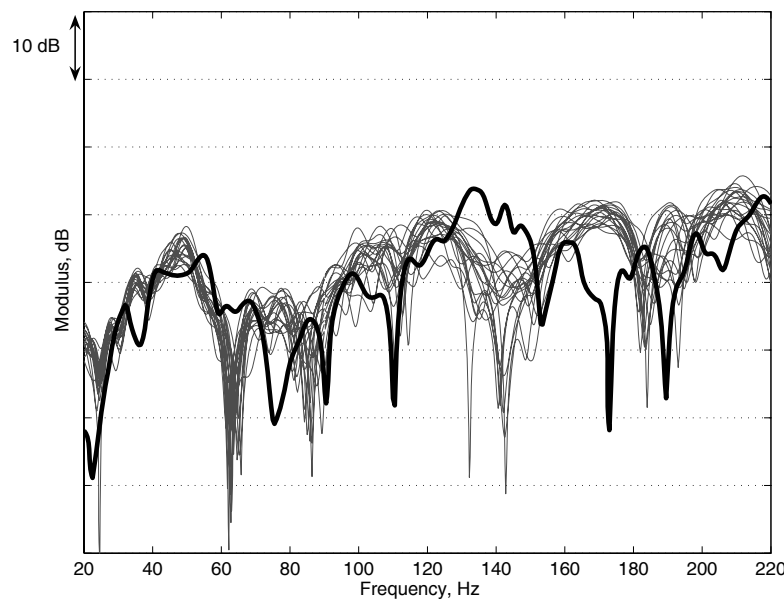

FIG. 12. Comparisons of the mean computational model results with the experiments. Graphs of the modulus in dB scale of the structural FRF for observation Obs6: experiments for the 20 cars (grey lines), mean computational model (thick solid line).

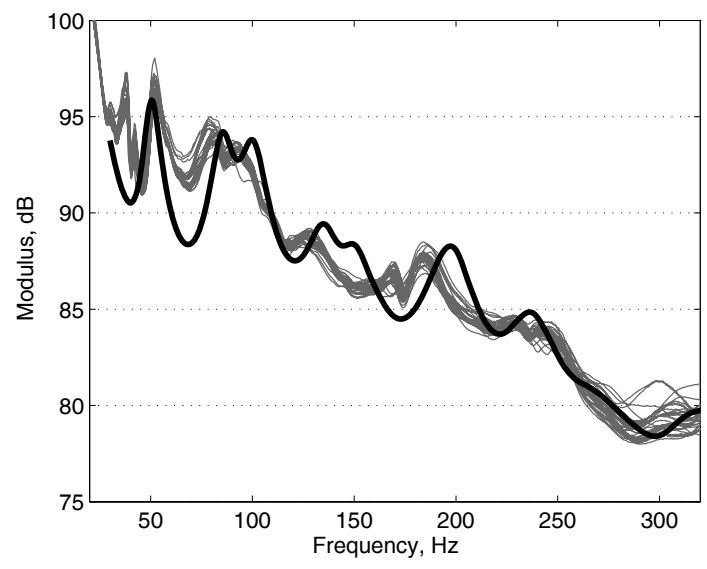

FIG. 13. Comparisons of the mean computational model results with the experiments. Graphs of the root mean square of the acoustic pressures averaged in the cavity in $\mathrm{dB}$ scale: experiments for the 30 configurations (grey lines), mean computational model (thick solid line).

this hypothesis allows the computational cost to be decreased. Secondly, different analyses have shown that the confidence regions are not very sensitive to the value of dispersion parameter $\delta_{D^{a}}$. This is due to the fact that the modal density is relatively high in the frequency band of analysis and in addition, the internal acoustic cavity has a large dissipative factor due to the presence of the absorbing materials as explained above. Finally, a sensitivity analysis of the responses has been carried out with respect to $\delta_{M^{a}}$ and $\delta_{K^{a}}$ and has shown that the confidence regions were very sensitive to the values of the dispersion parameters but that $\delta_{M^{a}}=\delta_{K^{a}}$ could be written.
FIG. 14. Comparisons of the mean computational model results with the experiments. Graphs of the modulus of the synthesized booming noise in $\mathrm{dB}[\mathrm{A}]$ scale: experiments for the 20 cars (grey lines), mean value of the experiments (thin solid line), mean computational model (thick solid line).

The method used to identify the dispersion parameter $\delta_{a}$ such that $\delta_{a}=\delta_{M^{a}}=\delta_{D^{a}}=\delta_{K^{a}}$ is the maximum likelihood method (Spall, 2003 ; Walter and Pronzato, 1997).

(2) The dispersion parameters $\delta_{M^{s}}, \delta_{D^{s}}$ and $\delta_{K^{s}}$ for the structure are identified using the structural frequency response functions (and not the structural-acoustic frequency response functions) of the experimental database defined in Subsection II.A. Therefore, the stochastic computational structural-acoustic model must be used. Then, for the identification of these dispersions parameters, dispersion parameters $\delta_{M^{a}}, \delta_{D^{a}}$ and $\delta_{K^{a}}$ are fixed to the identified experimental value $\delta_{a}$ and the value of $\delta_{C}$ is fixed to a nominal value. This last hypothesis is reasonable because the confidence regions of the random structural responses are not sensitive to the acoustic coupling (note that the structural acoustic responses are sensitive to $\delta_{C}$, but we are presently speaking about the identification of $\delta_{M^{s}}, \delta_{D^{s}}$ and $\delta_{K^{s}}$ using only the structural responses). Concerning $\delta_{D^{s}}$ the situation is similar to the case of the internal acoustic cavity, but for the structure, $\delta_{D^{s}}$ has been fixed to a nominal value which results from previous studies. In addition, it should be noted that the confidence regions of the structural responses are a little sensitive to $\delta_{D^{s}}$ compared with the high sensitivity of these responses with respect to $\delta_{M^{s}}$ and $\delta_{K^{s}}$. Concerning the experimental identification of $\delta_{M^{s}}$ and 
$\delta_{K^{s}}$ the assumption $\delta_{s}=\delta_{M^{s}}=\delta_{K^{s}}$ (similarly to the assumption used for the internal acoustic cavity) has been replaced by the following one $\delta_{s}=\sqrt{\delta_{M^{s}}^{2}+\delta_{K^{s}}^{2}}$. This choice results from computational tests and is much more efficient and more representative than the other one. For computational cost reasons (see below), the maximum likelihood method could not be used and consequently, has been substituted by the mean-square method with a differentiable objective function (Spall, 2003 ; Walter and Pronzato, 1997).

(3) For the identification of the dispersion parameter $\delta_{C}$ of the structural-acoustic coupling interface, the structural-acoustic frequency response functions of the experimental database defined in Subsection II.C are used. Therefore, the stochastic computational structural-acoustic model must be used. Then, for the identification of $\delta_{C}$, dispersion parameters $\delta_{M^{a}}, \delta_{D^{a}}, \delta_{K^{a}}$, $\delta_{M^{s}}, \delta_{D^{s}}$ and $\delta_{K^{s}}$ are fixed to their identified values (see above). For similar reasons to those given above, the maximum likelihood method cannot be used and should then be substituted by the mean-square method. Unfortunately, the corresponding objective function is not sufficiently sensitive to the value of $\delta_{C}$ and the maximum likelihood method is not efficient. So we have identified the value of $\delta_{C}$ using a trial method.

\section{Experimental identification of the dispersion parameters of the internal acoustic cavity}

As explained above, the maximum likelihood method is used to identify the dispersion parameter $\delta_{a}$ introduced in Subsection V.B(1). The random observation $Y$ and its corresponding experimental quantity $Y^{\exp }$ used for this identification is defined by

$$
Y=\int_{B_{f}} d B_{r m s}(f) d f \quad, \quad Y^{e x p}=\int_{B_{f}} d B_{r m s}^{\exp }(f) d f,
$$

in which $d B_{r m s}(f)$ corresponds to $d B_{r m s}^{e x p}(f)$ defined in Subsection II.B for the experiments and which is written as

$$
d B_{r m s}(f)=20 \log _{10}\left(\frac{\sqrt{\frac{1}{32} \sum_{\ell=1}^{32}\left|\boldsymbol{P}_{j \ell}^{a}(2 \pi f)\right|^{2}}}{P_{r e f}}\right),
$$

in which $j_{1}, \ldots, j_{32}$ are the DOFs corresponding to the measurement points. Let $Y^{\exp }\left(\eta_{1}\right), \ldots, Y^{\exp }\left(\eta_{30}\right)$ be the independent experimental realizations corresponding to the 30 configurations of the internal acoustic cavity. Let $\delta_{a} \mapsto \mathcal{L}\left(\delta_{a}\right)$ be the log-likelihood function defined by

$$
\mathcal{L}\left(\delta_{a}\right)=\sum_{\nu=1}^{30} \log _{10} p_{Y}\left(\delta_{a}, Y^{\exp }\left(\eta_{\nu}\right)\right)
$$

in which $y \mapsto p_{Y}\left(\delta_{a}, y\right)$ is the probability density function of random variable $Y$. For all fixed $\delta_{a}$, the righthand side of the above equation is estimated by the
Monte Carlo method with the stochastic computational structural-acoustic model presented in Subsection IV.A. The maximum likelihood method consists in solving the following optimization problem

$$
\delta_{a}^{o p t}=\arg \max _{\delta_{a}} \mathcal{L}\left(\delta_{a}\right)
$$

This method needs an accurate estimation of $p_{Y}\left(\delta_{a}, Y^{\exp }\left(\eta_{\nu}\right)\right)$ which has been estimated with 20,000 independent realizations for the Monte Carlo simulation. Instead of using an optimization algorithm to solve the above optimization problem, the values of the log-likelihood function have directly calculated in 8 values of $\delta_{a}$ in order to construct an approximation of its graph on the interval of interest. Fig. 15 displays the approximation of the graph of $\delta_{a} \mapsto \mathcal{L}\left(\delta_{a}\right)$ allowing the optimal value $\delta_{a}^{o p t}$ to be estimated. For this opti-

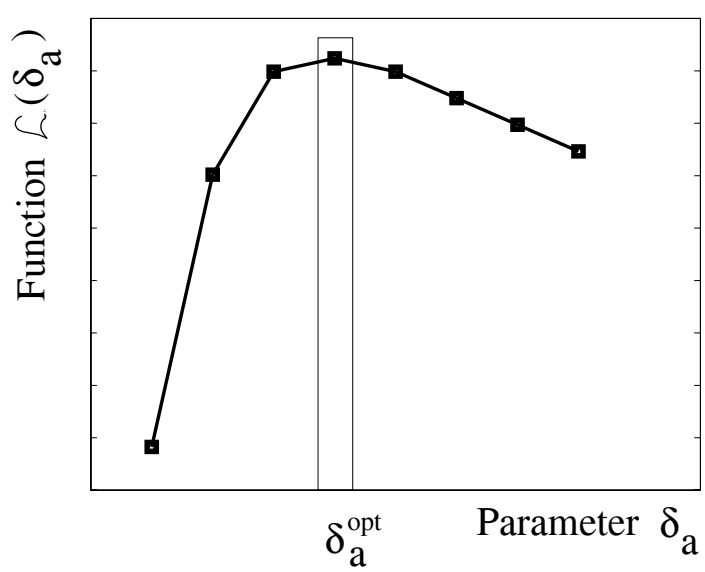

FIG. 15. Graph of $\delta_{a} \mapsto \mathcal{L}\left(\delta_{a}\right)$

mal value of $\delta_{a}^{o p t}$, Fig. 16 compares the experimental measurements with the computational results for the root mean square $d B_{r m s}^{\exp }(f)$ of the acoustic pressures averaged on the 32 microphones inside the internal acoustic cavity. In this figure, (1) the 30 grey lines represent the experimental measurements, (2) the upper and lower thick solid lines represent the upper and lower envelopes of the confidence region calculated for a probability level of 0.96 , (3) the mid thin solid line represents the mean value of the random response of the stochastic reduced computational model, and (4) the dashed line represents the response of the reduced mean computational model. It can be seen that the the experiments belongs to the confidence region with a probability level of 0.96 that validates the acoustic part of the stochatic computational model.

\section{Experimental identification of the dispersion parameters of the structure}

As explained in Subsection V.B(2), the mean-square method with differentiable objective function is used. 


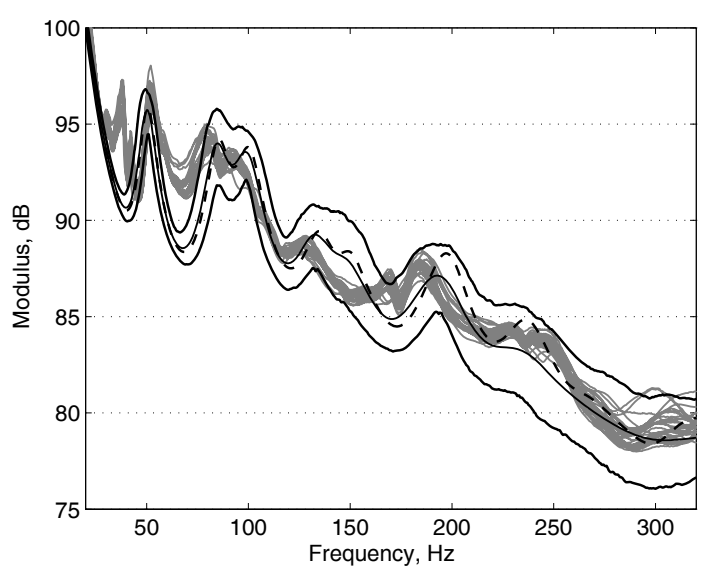

FIG. 16. Comparisons of the stochastic computational model results with the experiments. Graphs of the root mean square of the acoustic pressures averaged in the cavity in $\mathrm{dB}$ scale: experiments for the 30 configurations (grey lines), mean computational model (dashed line), mean value of the random response (mid thin solid line), confidence region: upper and lower envelopes are the upper and lower thick solid lines.

The dispersion parameters $\delta_{M^{a}}, \delta_{D^{a}}, \delta_{K^{a}}, \delta_{D^{s}}$ and $\delta_{C}$ are fixed to the values identified above (see V.B(1) and (2)). Consequently, we have to solve the optimization problem defined by

$$
\delta_{s}^{o p t}=\arg \min _{\delta_{s}} J\left(\delta_{s}\right),
$$

in which the objective function $J\left(\delta_{s}\right)$ depends only on $\delta_{s}$ and is defined by

$J\left(\delta_{s}\right)=2(1-\gamma)\|\| \boldsymbol{Z}\left(\delta_{s}\right)-\boldsymbol{m}\left(\delta_{s}\right) \mid\left\|^{2}+2 \gamma\right\| \boldsymbol{m}\left(\delta_{s}\right)-\underline{\boldsymbol{Z}}^{\exp } \|_{B}^{2}$,

with $\gamma=0.5$. In this equation, the random observation $\boldsymbol{Z}\left(\omega, \delta_{s}\right)=\left(Z_{1}\left(\omega, \delta_{s}\right), \ldots, Z_{6}\left(\omega, \delta_{s}\right)\right)$ of the stochastic computational model related to the six structural observations (see Subsection II.A) is such that

$$
Z_{j}\left(\omega, \delta_{s}\right)=\log _{10}\left(\omega^{2}\left|\boldsymbol{U}_{k_{j}}^{s}\left(\omega, \delta_{s}\right)\right|\right),
$$

and its mean value $\boldsymbol{m}\left(\omega, \delta_{s}\right)=\left(m_{1}\left(\omega, \delta_{s}\right), \ldots, m_{6}\left(\omega, \delta_{s}\right)\right)$ is

$$
\boldsymbol{m}\left(\omega, \delta_{s}\right)=E\left\{\boldsymbol{Z}\left(\omega, \delta_{s}\right)\right\} .
$$

The corresponding experimental observation is denoted by $\boldsymbol{Z}^{\exp }(\omega)=\left(Z_{1}^{\exp }(\omega), \ldots, Z_{6}^{\exp }(\omega)\right)$ and its mean value is

$$
\underline{Z}_{j}^{e x p}(\omega)=\frac{1}{20} \sum_{\ell=1}^{20} Z_{j}^{e x p}\left(\omega, \eta_{\ell}\right)
$$

Finally, the norms are defined by

$$
|| \boldsymbol{Z}\left(\delta_{s}\right)-\boldsymbol{m}\left(\delta_{s}\right)||^{2}=E\left\{\sum_{j=1}^{6} \int_{B}\left|Z_{j}\left(\omega, \delta_{s}\right)-m_{j}\left(\omega, \delta_{s}\right)\right|^{2} d \omega\right\}
$$

$\left\|\boldsymbol{m}\left(\delta_{s}\right)-\underline{\boldsymbol{Z}}^{\exp }\right\|_{B}^{2}=\sum_{j=1}^{6} \int_{B}\left|m_{j}\left(\omega, \delta_{s}\right)-\underline{Z}_{j}^{e x p}(\omega)\right|^{2} d \omega$

The first norm represents the variance of the computational model due to uncertainties and the second norm represents the bias between the experiments and the stochastic model. In this objective function, $B=2 \pi B_{f}$ with $B_{f}=[100,180] \mathrm{Hz}$ and the frequency resolution is $0.5 \mathrm{~Hz}$. For each evaluation of the objective function, the stochastic reduced computational model is solved using the Monte Carlo method with 1000 independent realizations and corresponding to a mean-square convergence of the second-order stochastic solution. Since each evaluation of the objective function requires about 500 hours of CPU time (the computations have been realized with 20 CPU yielding an elapsed time of 25 hours for each evaluation of the objective function), we have used a trial method consisting in computing the cost function for 10 values of the couple $\left(\delta_{M_{s}}, \delta_{K_{s}}\right)$. It can then be deduced the approximation of the graph of $\delta_{s} \mapsto J\left(\delta_{s}\right)$ for the 10 values of $\delta_{s}=\sqrt{\delta_{M_{s}}^{2}+\delta_{K_{s}}^{2}}$. Fig. 17 displays this approximation of the graph of $\delta_{s} \mapsto J\left(\delta_{s}\right)$ allowing the optimal value $\delta_{s}^{\text {opt }}$ to be estimated. From $\delta_{s}^{o p t}$, it can then be deduced the optimal values $\delta_{M_{s}}^{o p t}$ and $\delta_{K_{s}}^{o p t}$ of $\delta_{M_{s}}$ and $\delta_{K_{s}}$. For this optimal values $\delta_{M_{s}}^{o p t}$ and $\delta_{K_{s}}^{o p t}$ of $\delta_{M_{s}}$ and $\delta_{K_{s}}$, and

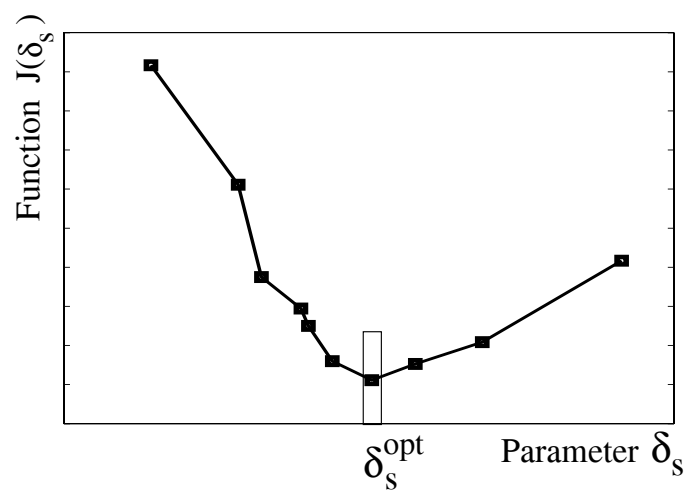

FIG. 17. Graph of $\delta_{s} \mapsto J\left(\delta_{s}\right)$

for the values of the other dispersion parameters fixed to their values previously identified, Figs. 18 and 19 compare the experimental measurements with the computational results for the moduli of the FRFs in $\mathrm{dB}$ concerning observations Obs4 (Fig. 18) and Obs6 (Fig. 19) and for the 20 cars. In these figures, (1) the 20 grey lines represent the experimental measurements, (2) the upper and lower thick solid lines represent the upper and lower envelopes of the confidence region calculated for a probability level of $0.96,(3)$ the mid thin solid line represents the mean value of the random response of the stochastic reduced computational model, and (4) the dashed line represents the response of the reduced mean computational model. It can be seen that the experiments belong to the confidence region with a probability level of 0.96 
that validates the structural part of the stochatic computational model.

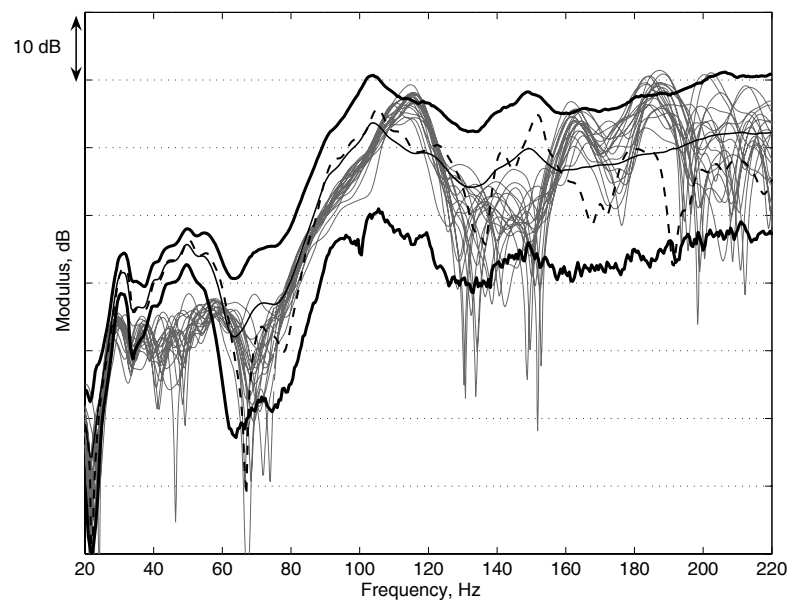

FIG. 18. Comparisons of the stochastic computational model results with the experiments for observation Obs4. Graphs of the moduli of the FRFs in dB scale: experiments for the 20 cars (grey lines), mean computational model (dashed line), mean value of the random response (mid thin solid line), confidence region: upper and lower envelopes are the upper and lower thick solid lines.

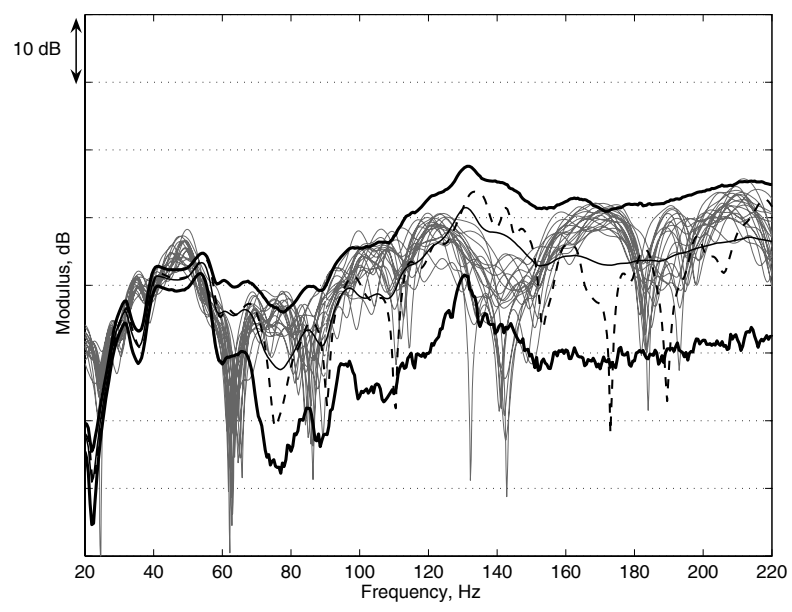

FIG. 19. Comparisons of the stochastic computational model results with the experiments for observation Obs6. Graphs of the moduli of the FRFs in $\mathrm{dB}$ scale: experiments for the 20 cars (grey lines), mean computational model (dashed line), mean value of the random response (mid thin solid line), confidence region: upper and lower envelopes are the upper and lower thick solid lines.

\section{E. Experimental validation of the stochastic computational structural-acoustic model for the booming noise}

In this subsection, we compare the experiments for the synthesized booming noise of the database (see Subsec- tion II.C) with the predictions given by the stochastic computational structural-acoustic model. For the calculation, the dispersion parameters $\delta_{M^{a}}, \delta_{D^{a}}, \delta_{K^{a}}, \delta_{M^{s}}$, $\delta_{D^{s}}, \delta_{K^{s}}$ and $\delta_{C}$ are fixed to their optimal values which are either fixed or experimentally identified as explained in the previous subsections. The Monte Carlo stochastic solver is used with 1500 realizations. A mean-square convergence analysis of the stochastic response has been carried out (Durand, 2007) and convergence is reached for this number of realizations. Fig. 20 compares the experimental measurements with the computational results for the booming noise in $\mathrm{dB}[\mathrm{A}]$ scale. The 20 thin grey lines represent the experimental measurements of the booming noise for the 20 cars and the thick grey line the mean value of the experiments. The upper and lower thick solid lines represent the upper and lower envelopes of the confidence region calculated for a probability level of 0.96 . The mid thin solid line represents the mean value of the random response of the stochastic reduced computational model. The mid dashed line represents the response of the reduced mean computational model. Taking into account the complexity of the vibroacoustic model, the obtained results validate the stochastic computational model and demonstrate its capability to predict experimental measurements knowing that the dispersion parameters of model uncertainties have been identified.

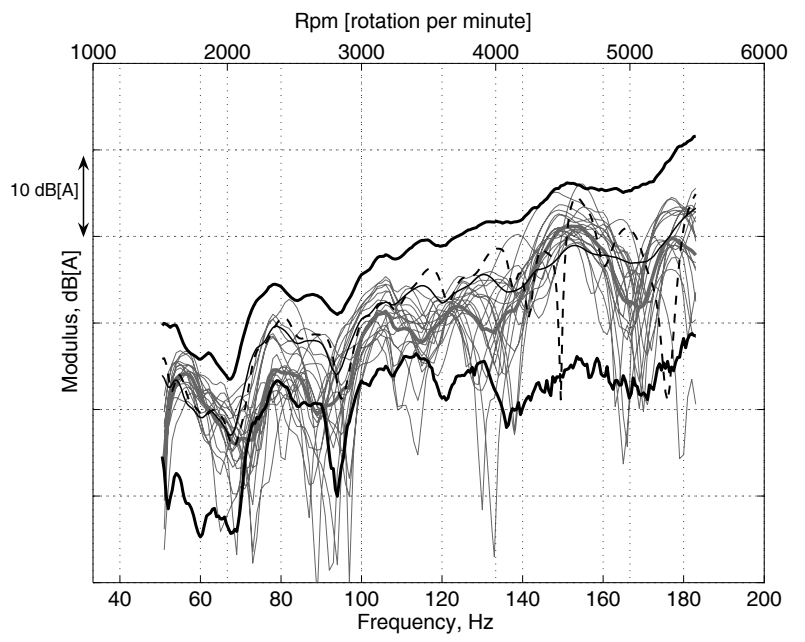

FIG. 20. Comparisons of the stochastic computational model results with the experiments for the booming noise. Graphs of the moduli of the FRFs in $\mathrm{dB}[\mathrm{A}]$ scale: experiments for the 20 cars (thin grey lines), mean value of the experiments (thich grey line), mean computational model (dashed line), mean value of the random response (mid thin solid line), confidence region: upper and lower envelopes are the upper and lower thick solid lines. 
In this paper, we have presented and validated a methodology to analyze a very complex structuralacoustic system in the low- and medium-frequency ranges. Both data uncertainties and model uncertainties have been taken into account in the computational model. The nonparametric probabilistic approach introduces a small number of dispersion parameters which can then be experimentally identified in solving optimization problems. In the context of the automative industry, a large experimental database has been constructed and has been presented in this paper. This database has been used for the identification of the probabilistic model. An experimental validation has been proposed for each step of the identification. The global experimental validation of the stochastic computational model has been obtained. The comparisons are good enough taking into account the complexity of the system and taking into account that the mean computational model is unique to represent all the variabilities induced by optional extras and manufacturing process. In addition, this mean computational model has voluntary not been updated with the measurements (this is the practical situation encountered by engineering in such an automative industry). With respect to the real structural-acoustic system, the dynamical behavior of the sound-proofing schemes (insulation materials) existing in the real cars (and in particular in the cars for which the experimental database has been constructed) has not been taken into account in the mean computational model (but has been taken into account as pure mass subsystems). Such a model could be improved in including such a dynamical model of the sound-proofing schemes.

\section{REFERENCES}

Attala, N., Hamdi, M.A., and Panneton, R. (2001). "Enhanced weak integral formulation for the mixed $(u, p)$ poroelastic equations," J. Acoust. Soc. Am. 109(6), 3065-3068.

Bathe, K.J. and Wilson, E.L. (1976) Numerical Methods in Finite Element Analysis, Prentice-Hall, Inc., Englewood Cliffs, New Jersey.

Durand, J.F., Gagliardini, L., and Soize, C. (2004). "Random modeling of frequency response functions of cars," International Conference on Modal Analysis Noise and Vibration Engineering (ISMA 2004) proceedings CDROM ISBN 90-73802-82-2.

Durand, J.F., Gagliardini, L., and Soize, C. (2005a). "Nonparametric modeling of the variability of vehicle structural-acoustic behavior," SAE 2005 Noise and Vibration Conference proceedings CDROM ISBN 0-76801657-6.

Durand, J.F., Gagliardini, L., and Soize, C. (2005b). "Nonparametric modeling of structural-acoustic coupling uncertainties," EuroDyn 2005 Conference proceedings
Durand, J.F. (2007). "Structural-acoustic modeling of automotive vehicles in presence of uncertainties and experimental identification and validation," PHD Thesis, Université de Marne-la-Vallée, France, 10 mai 2007.

Gagliardini, L., Houillon, L., Borello, G., and Petrinelli, L. (2005). "Virtual SEA-FEA-based modeling of mid-frequency structure borne noise," Sound and Vibration 39(1), 22-28

Géradin, M. and Rixen, D. (1994) Mechanical Vibrations, Wiley, Chichester, U.K.

Ghanem, R. and Spanos, P.D (2003). Stochastic Finite Elements: A spectral approach revised edition, Dover Publications, New-York.

Hamdi, M.A., Zhang, C., Mebarek, L., Anciant, M., and Mathieux, B. (2005). "Analysis of structuralacoustic performances of a fully trimmed vehicle using an innovative subsystem solving approach faciliting the cooperation between carmakers and sound-package suppliers," EuroDyn 2005 Conference proceedings CDROM ISBN 85-85769-03-03.

Hayashi, K., Yamaguchi, S., and Matsuda, A. (2000). "Analysis of booming noise in light-duty truck cab," J. of the Society of Automotive Engineers 21, 255-257.

Hills, E., Mace, B., and Ferguson, N.S. (2004). "Statistics of complex built-up structures," International Conference on Modal Analysis Noise and Vibration Engineering (ISMA 2004) proceedings CDROM ISBN 9073802-82-2.

Jaynes, E.T. (1957) "Information theory and statistical mechanics," Physical Review 106(4)620-630 and 108(2)171-190.

Kompella, M.S. and Bernhard, R.J (1996). "Variation of structural-acoustic characteristics of automotive vehicles," J. of Noise Control Engineering, 93-99.

Langley, R.S (2007). "On the diffuse field reciprocity relationship and vibrational energy variance in a random subsystem at high frequencies," J. Acoust. Soc. Am. $\mathbf{1 2 1}(2), 913-921$.

LeBot, A. (2002). "Energy transfer for high frequencies in built-up structures," J. Sound Vib. 250(2), 247275 .

Lighthill, J. (1978) Waves in Fluids, Cambridge University Press, MA.

Lyon, R.H. and Dejong, R.G (1995). "Theory and applications of Statistical Energy Analysis," edited by Butterworth-Heinemann, Boston.

Ohayon, R., and Soize, C. (1998). Structural Acoustics and Vibration, Academic Press, San Diego.

Pierce, A.D. (1989). Acoustics: An Introduction to its Physical Principles and Applications, Acoust. Soc. Am. Publications on Acoustics, Woodbury, NY, USA, (originally published in 1981, McGraw-Hill, New York).

Schueller, G.I (1997). "A state-of-the-art report on computational stochastic mechanics," Probab. Eng. Mech., 12(4), 197-321.

Schueller, G.I (2007). "On the treatment of uncertainties in structural mechanics and analysis," J. Comp. 
Struct., 85, 235-243.

Serfling, R .J. (1980). Approximation Theorems of Mathematical Statistics, John Wiley \& Sons.

Shannon, C.E. (1948). "A mathematical theory of communication," Bell System Technology Journal 27 379-423 and 623-659.

Shorter, P.J., and Langley R.S. (2005). "Vibroacoustic analysis of complex systems," J. Sound Vib. 288(3), 669-699.

Soize, C. (2000). "A nonparametric model of random uncertainties for reduced matrix models in structural dynamics," Probab. Eng. Mech., 15(3), 277-294.

Soize, C. (2001). "Maximum entropy approach for modeling random uncertainties in transient elastodynamics," J. Acoust. Soc. Am. 109(5), 1979-1996.

Soize, C. (2003). "Random matrix theory and nonparametric model of random uncertainties in vibration analysis," J. Sound Vib. 263, 893-916.

Soize, C. (2005a). "A comprehensive overview of a non-parametric probablistic approach of model uncertainties for predictive models in structural dynamics," J. Sound Vib. 288(3), 623-652.

Soize, C. (2005b). "Random matrix theory for model- ing random uncertainties in computational mechanics," Comp. Meth. Appl. Mech. Eng. 194(12-16), 1333-1366.

Sol, A., and Van-Herpe, F. (2001). "Numerical prediction of a whole car vibroacoustic behavior at low frequencies," SAE 2001 Noise and Vibration Conference proceedings CDROM ISBN 0-7680-0775-5.

Spall, J.C. (2003). Introduction to Stochastic Search and Optimization, John Wiley and Sons, Hoboken, New Jersey.

Sung, H.S., and Nefske, D.J. (2001). "Assessment of a vehicle concept finite element model for predicting structural vibration," SAE 2001 Noise and Vibration Conference proceedings CDROM ISBN 0-7680-0775-5.

Trusdell, C. (1960). "The Elements of Continuum Mechanics," Springer-Verlag, Berlin.

Walter, E., and Pronzato, L. (1997). Identification of Parametric Models from Experimental Data, Springer.

Wood, L.A., and Joachim, C.A (1987). "Interior noise scatter in four-cylinder sedans and wagons," Int. J. of Vehicle Design 8, 428-438.

Zienkiewicz, O. C., and Taylor, R. L. (2000). The Finite Element Method, Fifth edition, Vol. 1 to 3, Butterworth-Heinemann, Oxford. 